\title{
The influence of non-polar lipids on tear film dynamics
}

\author{
M. Bruna $\dagger$ and C. J. W. Breward \\ Mathematical Institute, University of Oxford, Oxford, OX2 6GG, U.K. \\ (Received ?; revised ?; accepted ?. - To be entered by editorial office)
}

In this paper we examine the effect that physiological non-polar lipids, residing on the surface of an aqueous tear film, have on the film evolution. In our model we track the evolution of the thickness of the non-polar lipid layer, the thickness of the aqueous layer and the concentration of polar lipids which reside at the interface between the two. We also utilise a force balance in the non-polar lipid layer in order to determine its velocity. We show how to obtain previous models in the literature from our model by making particular choices of the parameters. We see the formation of boundary layers in some of these submodels, across which the concentration of polar lipid and the non-polar lipid velocity and film thickness vary. We solve our model numerically for physically realistic parameter values, and we find that the evolution of the aqueous layer and the polar lipid layer are similar to that described by previous authors. However, there are interesting dynamics for the non-polar lipid layer. The effects of altering the key parameters are highlighted and discussed. In particular, we see that the Marangoni number plays a key role in determining how far over the eye the non-polar lipid spreads.

Key words: biomedical flows, thin films, capillary flows

\section{Introduction}

The tear film is a saline fluid, secreted by the lacrimal glands, which resides on the ocular surface of the eye. The film contributes to the smooth optical properties of the corneal surface, acts to moisten the ocular surface, protects the cornea and conjunctiva against bacterial contamination, eliminates foreign bodies and exfoliated cells from the ocular surface, and lubricates the motion of the eyelids.

Dry eye disease is a disorder of the tears and ocular surface which is associated with potential damage to the ocular surface. Dry eye results in increased osmolarity of the tear film which causes inflammation of the ocular surface (Lemp 2007). In severe cases, the tear film can rupture. There are two main types of dry eye (Lemp 2007): aqueous deficient dry eye (initiated, for example, by a lack of production of fluid by the lacrimal glands), and evaporative dry eye (caused by non-standard blinking or by an increase in the rate of evaporation of the tear film). Treatments for dry eye include replacement of tear fluid using artificial tears, wearing of goggles to reduce evaporation, and stimulation of tear production.

The tear film is inherently unstable and, after formation of a continuous layer by blinking, the tear film thins and will rupture unless the liquid is refreshed by another blink (Holly \& Lemp 1977). The tear film is estimated to be between 3 and $11 \mu \mathrm{m}$ thick (King-Smith et al. 2004) and was traditionally thought to be a three-layer structure (first

\footnotetext{
$\dagger$ Email address for correspondence: bruna@maths.ox.ac.uk
} 
described by Wolff 1946): an outer lipid layer containing a mixture of polar and non-polar components, an aqueous layer containing the bulk of the tear volume, and a mucous layer that resides on the corneal epithelium. Bron et al. (2004) note that this threelayer model is still generally accepted while in the review by Dieckow \& Argüeso (2012) they describe both the traditional model and a two-layer model in which the mucous and the aqueous layers comprise a single phase. Whether the mucins form a contiguous layer or are distributed throughout the aqueous layer remains a topic of debate amongst ophthalmologists, and henceforth we will assume they form part of the aqueous layer.

The aqueous component is between 3 and $11 \mu \mathrm{m}$ thick (see for example (King-Smith et al. 2004); we note that recent measurements are at the low-end of this range) and contains electrolytes, proteins, antimicrobials, mucins, and other molecules that serve to nourish and protect the ocular surface (Johnson \& Murphy 2004). The electrolytes present are responsible for the osmolarity of the tears. The aqueous layer is secreted from the lacrimal glands and fills the sacks under the upper and lower eyelids.

The lipid layer is between 40 and $80 \mathrm{~nm}$ thick, is secreted from the meibomian glands of the eyelids (located at the junction between hydrophilic and hydrophobic cells) and contains two classes of lipids: non-polar and polar (Chen et al. 2010). The lipid layer composition is an active and ongoing area of experimental research. The proportion of polar lipid in the meibum is small. Nicolaides et al. (1981) report that polar lipids account for up to $16 \%$ of the meibum, Tiffany (1978) reports that polar lipids account for up to $5 \%$ of the meibum, while a more recent study by Chen et al. (2010) reports polar free fatty acids forming $3 \%$ of the meibum. It is energetically favourable for the polar lipids to reside in contact with the aqueous layer, where they decrease the surface tension (to around $40-45 \mathrm{mN} / \mathrm{m}$ ) and enhance the stability of the film (Mudgil \& Millar 2011).

The non-polar lipids reside above the polar lipids (McCulley \& Shine 1997) and provide a barrier to evaporation of the aqueous component (McCulley \& Shine 2004). Both components are insoluble (Nagyova \& Tiffany 1999). The viscosity of the lipid mixture is reported to be 9.7-19.5 Pa.s (Tiffany \& Dart 1981), although this figure is considered to be unreliable by one of the authors (J. M. Tiffany 2009, personal communication) who has unpublished data suggesting that a viscosity of $0.1 \mathrm{~Pa} \cdot \mathrm{s}$ is a more reliable figure. The non-polar lipid provides a barrier to tear film evaporation: for normal tears, evaporation rates have been measured (Nichols et al. 2005) to be between 0.24 and $1.45 \mu \mathrm{m} / \mathrm{min}$ (note that these are mass transfer coefficients), while the evaporation rate increases significantly to $7 \mu \mathrm{m} / \mathrm{min}$ when the lipid layer is removed.

Blinking is fundamental to preserving a continuous tear film. During a blink, the upper eyelid moves down to meet the (almost stationary) lower eyelid, and then returns to the open position. During this motion, the tear film is mixed and redistributed back over the eye surface. The resulting tear film has menisci at each of the eyelids and a thin film over the ocular surface joining them. The upstroke of a blink takes approximately 200 milliseconds, the downstroke takes 100 milliseconds, and the interblink lasts between 4 and $10 \mathrm{~s}$. Blinks are classified as "complete" if the lids come into contact with each other or "partial" if they do not close completely. There are three types of blinking: spontaneous, reflex and voluntary (Kaufman et al. 2003). Spontaneous is the common blinking model and is the one considered in this paper. Reflex blinking is triggered by sensory stimulation and is associated with a high blink rate. Voluntary blinking is blinking initiated by the subject (in which the speed and/or interblink duration can be controlled). Blink velocity data have been recorded (Berke \& Mueller 1996; Jones et al. 2005) using high-speed cameras.

During a blink, some of the liquid in the sac under the upper eyelid is exchanged with liquid in the tear film. However, the average time for fluorescein solution to appear in the 
tear film when instilled under the lower eyelid is four minutes (MacDonald \& Maurice 1991), indicating that fluid transfer between the tear film and the liquid in the sac under the lower eyelid is negligible on the time scale of a single blink.

Following a blink, the non-polar lipid layer advances up the eye surface, as observed by Yokoi et al. (2008). The two-dimensional patterning of the non-polar lipid layer can persist between successive blinks. It is not possible to detect very thin layers of lipid, and so it is not clear from the experiments whether or not the non-polar lipid layer extends all the way across the eye, even by the end of the interblink.

There have been a number of previous models developed to describe various aspects of tear film evolution. Wong et al. (1996) formulate a coating model to explain the evolution of the thickness of a tear film during the interblink. The authors take account of the lipid layer by assuming that it renders the free surface tangentially immobile. The thinning of the tear film is simulated numerically, and it is shown that the minimum film thickness obeys a power law. They calculate a tear film breakup time, defined as the interval between the last complete blink and the first appearance of a rupture in the tear film, which agrees with those observed experimentally (Lemp 2007). Braun \& Fitt (2003) build on this model and develop a more complicated model for the interblink which includes the effects of evaporation and gravity. Albeit not the most dominant effects everywhere in the fluid domain, they are shown to affect the film drainage process.

Jones et al. (2005) adapt the model of Braun \& Fitt (2003) to incorporate the lipid layer in two other limits, which they term the active and inactive lipid limits. In the active lipid limit, the lipid layer is uniformly stretched between the upper and lower eyelids, while in the inactive lipid limit, there are no surface effects at all. They show that the eyelid velocity, the motion of the surface lipid layer and the storage of the tear film between blinks are of central importance in understanding the deposition of the tear film. Moreover, the effect of evaporation is found to be negligible over a typical relaxation period, reinforcing the results from Braun \& Fitt (2003).

In Jones et al. (2006), their previous model is extended to explicitly include the polar lipids during tear film deposition and the interblink. Their model includes a lubrication equation for the evolution of the film thickness, and a mass transport equation that describes the lipid concentration. Their model highlights the role of lipids in drawing the tear film up the cornea during the opening phase of the blink as well as the fact that nonuniform distributions of lipids may lead to a rapid thinning of the tear film behind the advancing lipid front, which is in agreement with the experimental data gathered. In a later article, Jones et al. (2008) focus on the eyelid and the tear film that pre-exists under the lid when the eye is closed. They apply standard lubrication theory to the fluid layer between the eyelid and ocular surface and couple this with an elastic mattress model for the soft tissue of the eyelid and ocular surface. This leads to a one-dimensional nonlinear partial differential equation governing the fluid pressure in the lubrication film, which is solved numerically by specifying a loading condition. The benefit of their model is that it allows predictions of factors which are known to be relevant to dry eye syndrome, namely the flux from under the upper eyelid, as well as normal and shear stresses acting on the ocular surface. They find that the pressure and shear stress under the eyelid act across a length of approximately $0.1 \mathrm{~mm}$, a value consistent with clinical observations.

Braun \& King-Smith (2007) address model problems for the tear film over multiple blink cycles in limits that yield a single lubrication equation for the tear film. For the lipid layer, they consider the same two cases as in Jones et al. (2005), namely (1) absence of naturally occurring lipid, and (2) polar lipid strongly affecting the surface tension. Solutions for multiple complete blink cycles are computed by considering the film on a sinusoidally varying domain with specified film thickness and volume flux at each 
end; only one end of the domain is moving, which is analogous to the upper eyelid moving with each blink. Heryudono et al. (2007) extend this work to model problems for tear films by implementing realistic lid motion from observed blinks. Their numerical computations are in agreement with in-vivo tear film thickness measurements under partial blink conditions.

By considering the limiting case where the lipid is strongly affecting the surface tension, Maki et al. (2008) implement an overset grid method to simulate the evolution of the tear film thickness during reflex tearing and compare the solutions to experimental observations. Their numerical simulations capture localised capillary thinning near the tear menisci, referred to as "black lines". Their computational results are in qualitative agreement with in vivo tear film thickness measurements.

Aydemir et al. (2011) employ a model for the aqueous layer and polar lipids similar to that in Jones et al. (2006), and include the opening phase as well as the interblink. The same pair of coupled differential equations for the tear film thickness and the polar lipid concentration are obtained and solved numerically. Their numerical results show that the polar lipid concentration alters the flux of tears over the surface of the eye, agreeing with the results found by Jones et al. (2006), and explain why polar lipid migrates up the eye even after the eyelids have stopped moving. The paper contains an asymptotic analysis of the model to explain the early time behaviour of the system. This model is used in Zubkov et al. (2012) as the basis for examining tear film osmolarity. In this paper, the salt concentration is tracked during blinking and the interblink, as well as during a saccade. The authors show that the osmolarity is highest where the tear film is thinnest (at the "black lines"), and that the rate of evaporation plays a key role in determining the osmolarity.

Evaporation from tear films has been considered only relatively recently in the literature. For example, in Li \& Braun (2012) a model for tear film dynamics and film temperature evolution during the interblink are considered, where the evaporative mass flux is related to changes in the pressure and temperature of the tear film. The model reproduces experimentally measured thinning rates once the temperature penetration depth has been fitted. Peng et al. (2014) study tear film breakup during the interblink and, in particular, examine the hypothesis that such a breakup occurs due to locally high evaporative thinning, caused by a prescribed reduction in the lipid layer thickness. Their one-dimensional model for the aqueous layer and salt concentration highlights the tear film parameters and external conditions when black spots and subsequent tear breakup may occur.

An excellent review of the modelling of the dynamics of tear films, summarising a number of these results, is presented in Braun (2012).

None of these models tracks the evolution of the non-polar lipid layer, which is crucial in preventing excessive evaporation of the aqueous layer and whose deficiencies are thought to be a driver for evaporative dry eye. Thus, in this article, we extend the model presented in Aydemir et al. (2011) to include both polar and non-polar lipids, with the aim of determining what (if any) influence the non-polar lipids have on the evolution of the tear film. The situation is similar to that presented in Matar et al. (2002). In that paper, the authors consider a two-layer system (pericellular liquid residing beneath a mucus layer) with an insoluble surfactant on the mucus-air interface. The structure of their model is the same as the one we will generate in this paper: involving evolution equations for the thicknesses of the two layers and the surfactant concentration, coupled with a force balance in the highly viscous upper layer. They neglect surface tension and evaporation, but include Van-der-Waals forces. They solve the problem numerically in several limits, on a static domain. 


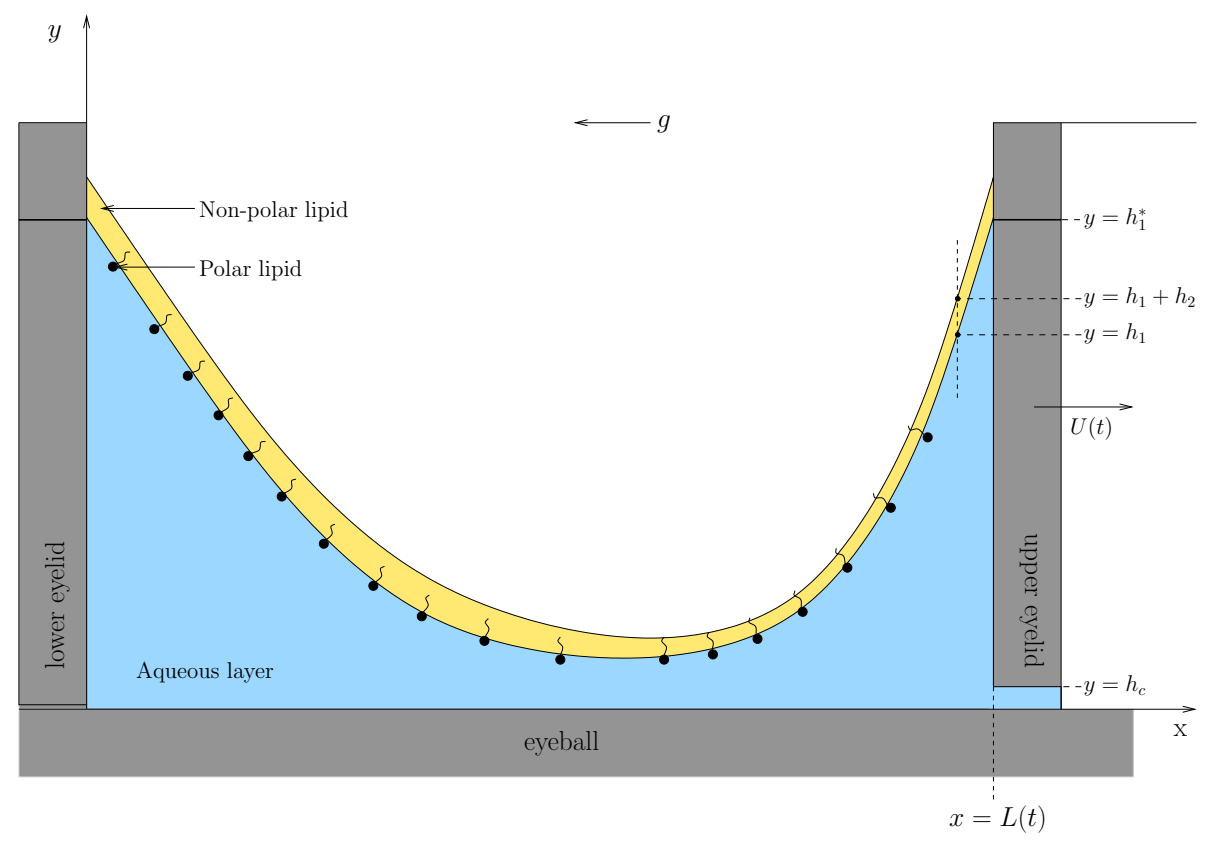

Figure 1. Schematic showing the geometry of the tear film.

In sections 2 and 3 we formulate the model and use the sizes of relevant non-dimensional parameter groups to reduce the model to four coupled equations for the thickness of the tear film, the thickness and the velocity of the non-polar lipid layer and the concentration of polar lipid residing at the interface between these two layers. In section 4 we present numerical simulations of the model and discuss the behaviour as various critical nondimensional parameter groups are varied. We then discuss in section 5 various asymptotic limits of the model that enable us to recover previous models in the literature. Finally, in section 6 we discuss our conclusions and suggest possible future investigations.

\section{Mathematical model for the evolution of the tear film}

In this section we derive our governing equations for the evolution of the tear film. We assume that the eyeball is flat (see argument in Aydemir et al. 2011), that the tear film is long and thin, and, for simplicity, that the flow is two dimensional, as shown in figure 1. We will utilise the slenderness of both the aqueous layer and the non-polar lipid layer to reduce the complexity of the model. We treat each of these layers as incompressible Newtonian viscous fluids, each with their own (constant) viscosity and density. We use subscript 1 to denote quantities in the aqueous layer, and subscript 2 to denote quantities in the non-polar lipid layer.

Our starting point is the two-dimensional Navier-Stokes equations (see Ockendon \& Ockendon 1995) for the each layer,

$$
\begin{gathered}
\nabla \cdot \mathbf{u}_{i}=0 \\
\rho_{i}\left(\mathbf{u}_{i t}+\left(\mathbf{u}_{i} \cdot \nabla\right) \mathbf{u}_{i}\right)=-\nabla p_{i}+\mu_{i} \nabla^{2} \mathbf{u}_{i}-\rho_{i} g \mathbf{i} .
\end{gathered}
$$

Here, in Cartesian coordinates, the fluid velocities are given by $\mathbf{u}_{i}=\left(u_{i}(x, y, t), v_{i}(x, y, t)\right)$, with pressures $p_{i}$, densities $\rho_{i}$, viscosities $\mu_{i}$ and gravity $g$ (acting in the $-\mathbf{i}$ direction, see figure 1). 
We assume that the polar lipid is constrained to reside at the interface, $y=h_{1}(x, t)$, between the aqueous and non-polar lipid layer and we denote its (surface) concentration by $\Gamma$, which evolves according to the advection-diffusion equation (Berger \& Corrsin 1974)

$$
\Gamma_{t}+\left(u_{s} \Gamma\right)_{S}+\kappa_{1} u_{n} \Gamma=D_{s} \Gamma_{S S},
$$

where $u_{s}$ and $u_{n}$ are the components of the surface velocity along the surface and normal to the surface respectively, $\kappa_{1}$ is the mean curvature of the surface and $D_{s}$ is the surface diffusivity. Subscript $S$ denotes differentiation along the surface.

We assume that the polar lipid acts in the same way as an insoluble surfactant, reducing the surface tension at the water/non-polar lipid interface. We follow Aydemir et al. (2011) and assume that the lipid is "dilute" and we use a linear equation of state, namely

$$
\gamma_{12}=\gamma_{12}^{*}-R T \Gamma,
$$

to relate the surface concentration of polar lipid to the surface tension, $\gamma_{12}$ of the interface. Here $\gamma_{12}^{*}$ is the surface tension when no polar lipid is present, $R$ is the universal gas constant and $T$ is the film temperature.

\subsection{Boundary conditions}

\subsubsection{Surface of the eye}

At the surface of the eye, we assume that there is no flux of water in or out, and that the mucous layer does not provide any slip. Thus we impose

$$
u_{1}=0, \quad v_{1}=0, \quad \text { at } y=0 .
$$

\subsubsection{Interface between aqueous layer and non-polar lipid layer}

We assume that the non-polar lipids form a continuous layer across the surface of the eye during the whole blink. (In the absence of experimental evidence, it is simpler to do this than to assume that there is a triple point that we have to track.) At the interface between the aqueous layer and the non-polar lipid layer, $y=h_{1}(x, t)$, we assume continuity of velocity, namely

$$
\mathbf{u}_{1}=\mathbf{u}_{2},
$$

Further, the motion of this interface is given by the kinematic condition, which, including evaporation of water with transfer coefficient $E(x, t)$, reads

$$
v_{1}=h_{1 t}+u_{1 s} h_{1 x}+E .
$$

Finally, at this interface we impose continuity of normal and tangential stress:

$$
\begin{gathered}
{\left[-p_{i}+2 \mu_{i} \frac{v_{i y}+u_{i x} h_{1 x}^{2}-h_{1 x}\left(u_{i y}+v_{i x}\right)}{1+h_{1 x}^{2}}\right]_{i=2}^{i=1}=\gamma_{12} \frac{h_{1 x x}}{\left(1+h_{1 x}^{2}\right)^{3 / 2}},} \\
{\left[\mu_{i} \frac{2\left(v_{i y}-u_{i x}\right) h_{1 x}+\left(u_{i y}+v_{i x}\right)\left(1-h_{1 x}^{2}\right)}{1+h_{1 x}^{2}}\right]_{i=2}^{i=1}=\frac{\gamma_{12 x}}{\sqrt{1+h_{1 x}^{2}}} .}
\end{gathered}
$$

\subsubsection{Interface between air and non-polar lipid}

At the interface between the air and the non-polar lipid $y=h(x, t)=h_{1}(x, t)+h_{2}(x, t)$, where $h_{2}(x, t)$ is the thickness of the non-polar lipid layer, we impose the kinematic condition

$$
v_{2}=h_{t}+u_{2 s} h_{x}+E,
$$


and continuity of normal and tangential stress

$$
\begin{aligned}
P_{0}-p_{2}+2 \mu_{2} \frac{v_{2 y}+u_{2 x} h_{x}^{2}-h_{x}\left(u_{2 y}+v_{2 x}\right)}{1+h_{x}^{2}} & =\gamma_{2 a i r} \frac{h_{x x}}{\left(1+h_{x}^{2}\right)^{3 / 2}}, \\
\mu_{2} \frac{2\left(v_{2 y}-u_{2 x}\right) h_{x}+\left(u_{2 y}+v_{2 x}\right)\left(1-h_{x}^{2}\right)}{1+h_{x}^{2}} & =0,
\end{aligned}
$$

where $P_{0}$ is the pressure in the air above the tear film and $\gamma_{2 a i r}$ is the surface tension of the air-non-polar lipid interface. Here we have assumed that the water which evaporates from the aqueous layer passes straight through the non-polar lipid layer and evaporates at the lipid-air interface at the same rate and does not alter the thickness of the non-polar lipid layer.

\subsubsection{Eyelids}

We suppose that the lower eyelid is stationary and the upper eyelid moves during a blink. We assume that the contact line between the aqueous layer and the eyelid is pinned at the mucocutaneous junction. We write

$$
h_{1}(0, t)=h_{1}^{*}=h_{1}(L, t),
$$

where $L(t)$ is the position of the upper eyelid. At the bottom eyelid, we assume that no water or lipid are supplied to the tear film, while at the upper lid we assume that that there is no lipid supplied, but that there is a fixed gap, $h_{c}$, between the bottom of the upper eyelid (see figure 1) and the surface of the eye, which delivers a flux of water. Given a Couette flow between the surface of the eye and the bottom of the eyelid, the flux is given by $\dot{L}(t) h_{c} / 2$. Denoting the cross-sectionally averaged flux of water by $q_{1}(x, t)$, the cross-sectionally averaged flux of non-polar lipid by $q_{2}(x, t)$ and the flux of polar lipid by $q_{s}(x, t)$, we write

$$
\begin{aligned}
& q_{1}(0, t)=0, \quad q_{s}(0, t)=0, \quad q_{2}(0, t)=0, \\
& q_{1}(L, t)=\left(h^{*}-\frac{h_{c}}{2}\right) \dot{L}, \quad q_{s}(L, t)=\Gamma(L, t) \dot{L}, \quad q_{2}(L, t)=h_{2}(L, t) \dot{L} .
\end{aligned}
$$

Finally, we have to prescribe two further boundary conditions on the non-polar lipid layer. Since the non-polar lipid wets the cells of the conjunctival epithelium, we should in principle prescribe a (small) contact angle between the non-polar lipid-air interface and the lid margin. However, having a small (or zero) contact angle is incompatible with the approach that we describe in section 3 , since we assume that slopes are small in order to utilise the lubrication approximation and derive the thin film equation. Indeed, we would only expect the contact angle to affect a change in the film thickness very close to the lid margins. We discuss how to correctly address the flow near this contact line in section 6 . Thus, we assume instead that the non-polar lipid-air interface approaches the lid margin at the same angle as the non-polar lipid-aqueous interface, and write

$$
h_{2 x}(0, t)=h_{2 x}(L, t)=0 .
$$

\subsection{Evaporation model}

We assume that the evaporative transfer rate, $E$, depends only on the thickness of the non-polar lipid layer. In practice, $E$ will also depend on the thickness of the aqueous layer, in that, as the aqueous layer becomes very thin, the evaporation rate will decrease due to the presence of the mucous layer (Zubkov et al. 2012). Our model for the evaporation is the simplest possible form that is consistent with the data described earlier; 

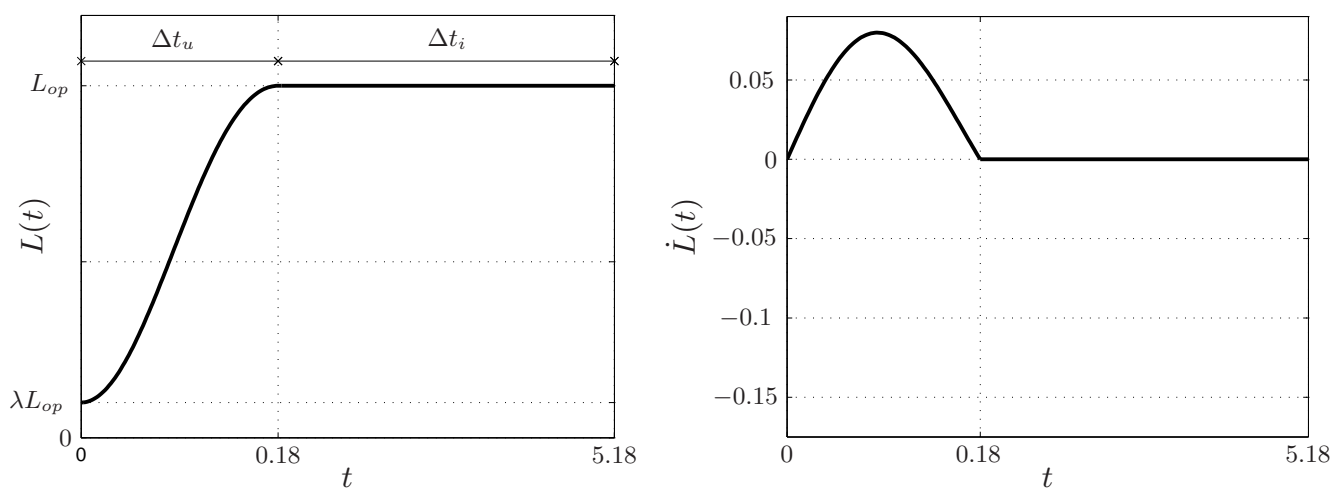

FiguRE 2. (a) Upper lid position $L(t)$ given by (2.15) and (b) corresponding velocity $\dot{L}(t)$ during the upstroke and interblink. The parameter values are $\Delta t_{u}=0.1758 \mathrm{~s}, \Delta t_{i}=5 \mathrm{~s}, L_{o p}=0.01 \mathrm{~m}$ and $\lambda=0.1$. Note that the $t$-axis is not to scale.

the evaporation rate increases as the amount of lipid decreases, namely

$$
E\left(h_{2}\right)=\frac{E_{0}}{\nu+h_{2}},
$$

where $E_{0}$ and $\nu$ are evaporation constants, and $E_{0} / \nu$ is the rate of evaporation when there is no non-polar lipid layer.

As noted earlier, we know that $E=7 \mu \mathrm{m} / \mathrm{min}$ when $h_{2}=0$ and we assume that $E=0.3 \mu \mathrm{m} / \mathrm{min}$ when $h_{2}=1.5 \times 10^{-8} \mathrm{~m}$, which is consistent with the data in Nichols et al. (2005). We then back out that, $E_{0}=7.84 \times 10^{-17} \mathrm{~m}^{2} / \mathrm{s}$ and $\nu=6.72 \times 10^{-10} \mathrm{~m}$. Of course, we could have picked other pairs of $E$ and $h_{2}$; we have checked with several other combinations that the results are qualitatively the same.

\subsection{Upper lid motion model}

We are interested in studying the tear film evolution over a blink cycle, consisting of the opening phase or upstroke (from lids closest together to eye fully open), the interblink period and the closing phase or downstroke. However, in this paper we restrict ourselves to the upstroke and the interblink. We suppose that the lids are not completely closed at the start of the blink: there is a fraction $\lambda$ of the fully open domain $\left[0, L_{o p}\right]$ which is exposed when the lids are in a closed position.

To describe the upper lid motion, and hence the moving domain $x \in[0, L(t)]$, we use the model proposed by Berke \& Mueller (1998) modified to allows us to define partial blinks as in Braun \& King-Smith (2007). From Berke \& Mueller (1998), the upstroke duration is $\Delta t_{u}=175.8 \mathrm{~ms}$ and the maximum upstroke velocity is $V_{\max }=9.1 \mathrm{~cm} / \mathrm{s}$ corresponding to a complete blink $(\lambda=0)$.

The upper lid position moves between $\lambda L_{o p} \leqslant L(t) \leqslant L_{o p}$ during $\Delta t_{u}$ for the upstroke while it remains fixed $L(t)=L_{o p}$ during $\Delta t_{i}$ corresponding to the interblink. In particular, the upper lid position is given by

$$
L(t)= \begin{cases}\lambda L_{o p}+(1-\lambda) L_{o p}\left(\frac{t}{\Delta t_{u}}\right)^{2} \exp \left[1-\left(\frac{t}{\Delta t_{u}}\right)^{2}\right], & 0 \leqslant t \leqslant \Delta t_{u}, \\ L_{o p}, & \Delta t_{u} \leqslant t \leqslant \Delta t_{u}+\Delta t_{i} .\end{cases}
$$

The velocity of the upper lid $\dot{L}(t)$ is obtained by differentiating (2.15). Figure 2 shows the lid position $L(t)$ and velocity $\dot{L}(t)$ over a blink cycle. 


\subsection{Initial conditions}

In addition to assuming that the eyelids start a distance $L_{c l}=\lambda L_{o p}$ apart, we suppose that the polar lipid has constant concentration and the non-polar lipids have constant thickness before a blink. Thus we write

$$
L=L_{c l}, \quad \Gamma=\Gamma^{*}, \quad h_{2}=h_{2}^{*} \quad \text { at } \quad t=0 .
$$

Finally, we assume that the shape of the water-lipid interface is determined by capillary statics, given a particular aqueous volume, and thus reads

$$
h_{1}=\frac{3 V}{2 \lambda L_{o p}}-\frac{h_{1}^{*}}{2}+\frac{3}{2}\left(h_{1}^{*}-\frac{V}{\lambda L_{o p}}\right)\left(\frac{2 x}{\lambda L_{o p}}-1\right)^{2},
$$

where $V$ is the initial volume per unit length across the eye. We estimate $V$ as follows. The total exposed volume when the eye is open is divided by the width of the eye, and then the volume of water exposed as the eyelid is drawn back is taken away to give $V$.

\section{Non-dimensional model}

We non-dimensionalise the governing equations and boundary conditions, informed by the following facts:

- The distance between the eyelids varies from $L_{c l}$ when the eye is closed to $L_{o p}$ when the eye is completely open. We take $L_{o p}$ as the characteristic lengthscale of the problem. The characteristic thickness of the film varies across the eye, and is largest in the menisci, and smallest in regions near to each menisci called "black lines". We choose to scale thicknesses with the aqueous layer thickness at the lid margins, $h_{1}^{*}$, so that the aspect ratio is

$$
\epsilon=\frac{h_{1}^{*}}{L_{o p}} \ll 1 .
$$

We note that this is the largest that the aspect ratio can be. If we had taken a typical thickness in the middle of the film, $\epsilon$ would have been much smaller.

- The non-polar lipid layer is always much thinner than the aqueous layer, with initial characteristic non-polar lipid thickness $h_{2}^{*}$. We define $\delta$ to be the ratio of the layer thicknesses, namely

$$
\delta=\frac{h_{2}^{*}}{h_{1}^{*}} \ll 1 .
$$

- The viscosity of the non-polar lipid layer is much larger than the viscosity of the aqueous layer. We define $\mu^{*}$ to be the viscosity ratio, and we have

$$
\mu^{*}=\frac{\mu_{2}}{\mu_{1}} \gg 1 .
$$

- In order to keep the pressure at leading order in both the aqueous and non-polar lipid layers in anticipation that one will be a lubrication flow and one will be an extensional flow, we use different pressure scales $P_{i}$ in each layer:

$$
P_{1}-P_{0}=\frac{\mu_{1} U}{\epsilon^{2} L_{o p}}, \quad P_{2}-P_{0}=\frac{\mu_{2} U}{L_{o p}},
$$

where $P_{0}$ is the constant pressure in the air, and $U$ is the horizontal characteristic velocity (identical in both layers). Note that these pressures are the same order if $\mu_{1} / \mu_{2}=O\left(\epsilon^{2}\right)$. 
Since the lipid layer is much thinner than the aqueous layer, we introduce a new coordinate $\zeta=y-h_{1}(x, t)$ for measuring distance through the non-polar lipid layer. We non-dimensionalise the model using:

$$
\begin{array}{lll}
x=L_{o p} x^{\prime}, & L=L_{o p} L^{\prime}, & E=\frac{E_{0}}{\nu} E^{\prime}, \\
y=\epsilon L_{o p} y^{\prime}, & h=\epsilon L_{o p} h^{\prime}, & h_{1}=\epsilon L_{o p} h_{1}^{\prime}, \\
\zeta=\delta \epsilon L_{o p} \zeta^{\prime}, & h_{2}=\delta \epsilon L_{o p} h_{2}^{\prime}, & t=\frac{L_{o p}}{U} t^{\prime}, \\
u_{i}=U u_{i}^{\prime}, & v_{i}=\epsilon U v_{i}^{\prime}, & \Gamma=\Gamma^{*} \Gamma^{\prime}, \\
\gamma_{12}=\gamma_{12}^{*}-R T \Gamma^{\prime}, & p_{1}=P_{0}+\frac{\mu_{1} U}{\epsilon^{2} L_{o p}} p_{1}^{\prime}, & p_{2}=P_{0}+\frac{\mu_{2} U}{L_{o p}} p_{2}^{\prime},
\end{array}
$$

where $U$ is the characteristic flow speed, which we choose (following Aydemir et al. 2011) as the average speed during the opening phase of the blink. We note that $u_{s}$ and $u_{n}$ scale like $u_{1}$ and $v_{1}$ respectively. Dropping the primes in the notation, the non-dimensionalised equations in the aqueous layer are

$$
\begin{aligned}
u_{1 x}+v_{1 y} & =0 \\
\epsilon^{2} \operatorname{Re}_{1}\left(u_{1 t}+u_{1} u_{1 x}+v_{1} u_{1 y}\right) & =-p_{1 x}+\epsilon^{2} u_{1 x x}+u_{1 y y}-\epsilon^{2} S t_{1}, \\
\epsilon^{4} R e_{1}\left(v_{1 t}+u_{1} v_{1 x}+v_{1} v_{1 y}\right) & =-p_{1 y}+\epsilon^{4} v_{1 x x}+\epsilon^{2} v_{1 y y}
\end{aligned}
$$

where $R e_{1}=\rho_{1} U L_{o p} / \mu_{1}$ and $S t_{1}=\rho_{1} g L_{o p}^{2} / \mu_{1} U$ are the Reynolds and Stokes numbers in the aqueous layer respectively, while in the non-polar lipid layer, we have

$$
\begin{gathered}
\delta u_{2 x}-h_{1 x} u_{2 \zeta}+v_{2 \zeta}=0 \\
\delta \epsilon^{2} R e_{2}\left[\delta u_{2 t}+u_{2}\left(\delta u_{2 x}-h_{1 x} u_{2 \zeta}\right)+v_{2} u_{2 \zeta}\right]=-\delta \epsilon^{2}\left(\delta p_{2 x}-h_{1 x} p_{2 \zeta}\right)+u_{2 \zeta \zeta} \\
+\epsilon^{2}\left(\delta^{2} u_{2 x x}-2 \delta h_{1 x} u_{2 x \zeta}-\delta h_{1 x x} u_{2 \zeta}+h_{1 x}^{2} u_{2 \zeta \zeta}\right)-\delta^{2} \epsilon^{2} S t_{2} \\
\delta \epsilon^{4} \operatorname{Re}_{2}\left[\delta v_{2 t}+u_{2}\left(\delta v_{2 x}-h_{1 x} v_{2 \zeta}\right)+v_{2} v_{2 \zeta}\right]=-\delta p_{2 \zeta}+v_{2 \zeta \zeta} \\
+\epsilon^{2}\left(\delta^{2} v_{2 x x}-2 \delta h_{1 x} v_{2 x \zeta}-\delta h_{1 x x} v_{2 \zeta}+h_{1 x}^{2} v_{2 \zeta \zeta}\right)
\end{gathered}
$$

where $R e_{2}=\rho_{2} U L_{o p} / \mu_{2}$ and $S t_{2}=\rho_{2} g L_{o p}^{2} / \mu_{2} U$ are the Reynolds and Stokes numbers in the non-polar lipid layer respectively.

The non-dimensionalised equation for the concentration of polar lipid reads

$$
\left(1+\epsilon^{2} h_{1 x}^{2}\right) \Gamma_{t}+\sqrt{1+\epsilon^{2} h_{1 x}^{2}}\left(\frac{u_{1}+\epsilon^{2} v_{1} h_{1 x}}{\sqrt{1+\epsilon^{2} h_{1 x}^{2}}} \Gamma\right)_{x}+\epsilon^{2} h_{1 x x} \frac{v_{1}-h_{1 x} u_{1}}{1+\epsilon^{2} h_{1 x}^{2}} \Gamma=\frac{\Gamma_{x x}}{P e},
$$

where $P e=U L_{o p} / D_{s}$ is the surface Péclet number.

\subsection{Non-dimensionalised boundary conditions}

At the eye surface, we have

$$
u_{1}=v_{1}=0, \quad y=0 .
$$

At the water-non-polar lipid interface, we have

$$
u_{1}=u_{2}, \quad v_{1}=v_{2}, \quad v_{1}=h_{1 t}+u_{1 s} h_{1 x}+\mathcal{E} E,
$$


where $\mathcal{E}=E_{0} / \epsilon U \nu$ is the evaporation number,

$$
\begin{aligned}
\epsilon^{2} \mu^{*} p_{2} & -p_{1}+2 \epsilon^{2}\left[\frac{v_{1 y}+\epsilon^{2} h_{1 x}^{2} u_{1 x}-h_{1 x}\left(u_{1 y}+\epsilon^{2} v_{1 x}\right)}{1+\epsilon^{2} h_{1 x}^{2}}\right] \\
& -\frac{2 \epsilon^{2} \mu^{*}}{\delta}\left\{\frac{v_{2 \zeta}+\epsilon^{2} h_{1 x}^{2}\left(\delta u_{2 x}-h_{1 x} u_{2 \zeta}\right)-h_{1 x}\left[u_{2 \zeta}+\epsilon^{2}\left(\delta v_{2 x}-h_{1 x} v_{2 \zeta}\right)\right]}{1+\epsilon^{2} h_{1 x}^{2}}\right\} \\
& =\left(\frac{\epsilon^{3}}{C a_{1}}-\epsilon^{3} M a \Gamma\right) \frac{h_{1 x x}}{\left(1+\epsilon^{2} h_{1 x}^{2}\right)^{3 / 2}},
\end{aligned}
$$

where $C a_{1}=\mu_{1} U / \gamma_{12}^{*}$ is the water-lipid capillary number and $M a=R T \Gamma^{*} / \mu_{1} U$ is the Marangoni number, and

$$
\begin{aligned}
& {\left[\frac{2 \epsilon^{2} h_{1 x}\left(v_{1 y}-u_{1 x}\right)+\left(1-\epsilon^{2} h_{1 x}^{2}\right)\left(u_{1 y}+\epsilon^{2} v_{1 x}\right)}{1+\epsilon^{2} h_{1 x}^{2}}\right]} \\
& -\frac{\mu^{*}}{\delta}\left\{\frac{2 \epsilon^{2} h_{1 x}\left(v_{2 \zeta}-\delta u_{2 x}+h_{1 x} u_{2 \zeta}\right)+\left(1-\epsilon^{2} h_{1 x}^{2}\right)\left[u_{2 \zeta}+\epsilon^{2}\left(\delta v_{2 x}-h_{1 x} v_{2 \zeta}\right)\right]}{1+\epsilon^{2} h_{1 x}^{2}}\right\} \\
& =\frac{-\epsilon M a \Gamma_{x}}{\left(1+\epsilon^{2} h_{1 x}^{2}\right)^{1 / 2}} .
\end{aligned}
$$

At the air-polar lipid interface, we have

$$
h_{t}+u_{2 s} h_{x}+\mathcal{E} E=v_{2},
$$

$$
\begin{aligned}
\frac{\delta \epsilon}{C a_{2}} & \frac{h_{x x}}{\left(1+\epsilon^{2} h_{x}^{2}\right)^{3 / 2}} \\
& =-\delta p_{2}+2\left\{\frac{v_{2 \zeta}+\epsilon^{2} h_{x}^{2}\left(\delta u_{2 x}-h_{1 x} u_{2 \zeta}\right)-h_{x}\left[u_{2 \zeta}+\epsilon^{2}\left(\delta v_{2 x}-h_{1 x} v_{2 \zeta}\right)\right]}{1+\epsilon^{2} h_{1 x}^{2}}\right\},
\end{aligned}
$$

where $C a_{2}=\mu_{2} U / \gamma_{2 a i r}$ is the lipid-air capillary number, and

$$
2 \epsilon^{2} h_{x}\left(v_{2 \zeta}-\delta u_{2 x}+h_{1 x} u_{2 \zeta}\right)+\left(1-\epsilon^{2} h_{x}^{2}\right)\left[u_{2 \zeta}+\epsilon^{2}\left(\delta v_{2 x}-h_{1 x} v_{2 \zeta}\right)\right]=0
$$

The non-dimensionalised evaporation rate is given by

$$
E\left(h_{2}\right)=\frac{\hat{\nu}}{\hat{\nu}+h_{2}},
$$

where $\hat{\nu}=\nu / \delta \epsilon L_{o p}$.

The boundary conditions at the eyelids read

$$
\begin{aligned}
& h_{1}=1, \quad h_{2 x}=0, \quad q_{1}=0, \quad q_{2}=0, \quad q_{s}=0 \quad \text { at } \quad x=0, \\
& h_{1}=1, \quad h_{2 x}=0, \quad q_{1}=\chi \dot{L}, \quad q_{2}=h_{2} \dot{L}, \quad q_{s}=\Gamma \dot{L} \quad \text { at } \quad x=L,
\end{aligned}
$$

where $\chi=\left(1-h_{c} / 2 \epsilon L_{o p}\right)$ The position of the upper eyelid is given by

$$
L(t)= \begin{cases}\lambda+(1-\lambda)\left(\frac{t}{\Delta \hat{t}_{u}}\right)^{2} \exp \left[1-\left(\frac{t}{\Delta \hat{t}_{u}}\right)^{2}\right], & 0 \leqslant t \leqslant \Delta \hat{t}_{u}, \\ 1, & \Delta \hat{t}_{u} \leqslant t \leqslant \Delta \hat{t}_{u}+\Delta \hat{t}_{i},\end{cases}
$$

where $\Delta \hat{t}_{u}=\Delta t_{u} U / L_{o p}$ and similarly for $\Delta \hat{t}_{i}$. Finally, we find that the initial conditions become

$$
\Gamma=1, \quad h_{2}=1, \quad h_{1}=\frac{3 \hat{V}}{2}-\frac{1}{2}+\frac{3}{2}(1-\hat{V})\left(\frac{2 x}{\lambda}-1\right)^{2},
$$

where $\hat{V}=V / \epsilon \lambda L_{o p}^{2}$. 


\begin{tabular}{|c|c|c|c|}
\hline Description & Parameter & Value & Units \\
\hline Aqueous layer density & $\rho_{1}$ & $10^{3}$ & $\mathrm{~kg} / \mathrm{m}^{3}$ \\
\hline Aqueous layer viscosity & $\mu_{1}$ & $1.3 \cdot 10^{-3}$ & $\mathrm{~Pa} \cdot \mathrm{s}$ \\
\hline Surface tension aqueous-lipid interface & $\gamma_{12}^{*}$ & $4.5 \cdot 10^{-2}$ & $\mathrm{~N} / \mathrm{m}$ \\
\hline Aqueous layer thickness at eyelids & $h_{1}^{*}$ & $2.5 \cdot 10^{-4}$ & $\mathrm{~m}$ \\
\hline Aqueous layer total volume & $V_{\text {tot }}$ & $5 \cdot 10^{-9}$ & $\mathrm{~m}^{3}$ \\
\hline Aqueous layer initial volume per unit length & $V$ & $2.5 \cdot 10^{-7}$ & $\mathrm{~m}^{2}$ \\
\hline Evaporation constant & $E_{0}$ & $7.84 \cdot 10^{-17}$ & $\mathrm{~m}^{2} / \mathrm{s}$ \\
\hline Evaporation constant & $\nu$ & $6.72 \cdot 10^{-10}$ & $\mathrm{~m}$ \\
\hline Non-polar lipid layer density & $\rho_{2}$ & $9 \cdot 10^{2}$ & $\mathrm{~kg} / \mathrm{m}^{3}$ \\
\hline Non-polar lipid layer viscosity & $\mu_{2}$ & 0.1 & $\mathrm{~Pa} \cdot \mathrm{s}$ \\
\hline Non-polar lipid layer initial thickness & $h_{2}^{*}$ & $3 \cdot 10^{-7}$ & $\mathrm{~m}$ \\
\hline Surface tension lipid-air interface & $\gamma_{2 a i r}$ & $1.8 \cdot 10^{-2}$ & $\mathrm{~N} / \mathrm{m}$ \\
\hline Polar lipid surface diffusion & $D_{s}$ & $3 \cdot 10^{-8}$ & $\mathrm{~m}^{2} / \mathrm{s}$ \\
\hline Polar lipid concentration & $\Gamma^{*}$ & $4 \cdot 10^{-7}$ & $\mathrm{~mol} / \mathrm{m}^{2}$ \\
\hline Distance between opened lids & $L_{o p}$ & $10^{-2}$ & $\mathrm{~m}$ \\
\hline Distance between closed lids & $\lambda L_{o p}$ & $10^{-3}$ & $\mathrm{~m}$ \\
\hline Film under the upper lid thickness & $h_{c}$ & $5 \cdot 10^{-6}$ & $\mathrm{~m}$ \\
\hline Average upper eyelid upstroke velocity & $U$ & $5.1 \cdot 10^{-2}$ & $\mathrm{~m} / \mathrm{s}$ \\
\hline
\end{tabular}

TABLE 1. Typical dimensions and properties for the tear film.

\subsection{Parameters}

In this section we summarise all the parameters introduced in the formulation of the tear film model. Table 1 lists the dimensional quantities, while estimates for the dimensionless parameters are given in table 2 .

\subsection{Distinguished parameter limit}

We discuss our assumptions about the sizes of the dimensionless groups. Our aim here is to establish a relevant distinguished limit of the model, which we can simplify to a sublimit using the actual parameter values given in table 2. Our primary focus is on the interplay between the surface tension forces and the two layers during the interblink, rather than inertial effects during the upstroke. Thus, despite the size of both of the Reynolds numbers, and in keeping with the rest of the mathematical literature on tear films, we choose upfront to ignore inertia in both layers from this point onwards. We will see later that the fluid velocity during the interblink is much smaller than during the blink so this assumption will be particularly valid in the interblink period. Our assumptions are as follows:

- The aspect ratio of the aqueous layer, $\epsilon$, is small. We compare all other parameters to $\epsilon$ when determining their size. 


\begin{tabular}{llc}
\hline Parameter & Description & Value \\
\hline$\chi=\left(1-h_{c} / 2 \epsilon L_{o p}\right)$ & Parameter controlling flux under upper eyelid & 0.99 \\
$\hat{V}=V / \epsilon \lambda L_{o p}^{2}$ & Aqueous layer initial volume per unit width & 0.99 \\
$\lambda$ & Initial fraction of exposed eye domain & 0.1 \\
$\Delta \hat{t}_{u}=\Delta t_{u} U / L_{o p}$ & Duration of upstroke & 0.9 \\
$\Delta \hat{t}_{i}=\Delta t_{i} U / L_{o p}$ & Duration of interblink & 25.6 \\
$\epsilon=h_{1}^{*} / L_{o p}$ & Tear film aspect ratio & $2.5 \cdot 10^{-2}$ \\
$\delta=h_{2}^{*} / h_{1}^{*}$ & Aqueous-lipid thicknesses ratio & $1.2 \cdot 10^{-3}$ \\
$R e_{1}=\rho_{1} U L_{o p} / \mu_{1}$ & Aqueous layer Reynolds number & $3.9 \cdot 10^{2}$ \\
$R e_{2}=\rho_{2} U L_{o p} / \mu_{2}$ & Non-polar lipid layer Reynolds number & 4.6 \\
$\mathcal{C}_{1}=\epsilon^{3} \gamma_{12}^{*} / \mu_{1} U$ & Aqueous-lipid interface Capillary number & $1.1 \cdot 10^{-2}$ \\
$\mathcal{C}_{2}=\epsilon \gamma_{2 a i r} / \mu_{2} U$ & Lipid-air interface Capillary number & $8.6 \cdot 10^{-2}$ \\
$\mathcal{S}_{1}=\epsilon^{2} \rho_{1} g L_{o p}^{2} / \mu_{1} U$ & Aqueous-layer Stokes number & 9.4 \\
$\mathcal{S}_{2}=\rho_{2} g L_{o p}^{2} / \mu_{2} U$ & Non-polar lipid layer Stokes number & 172 \\
$\mathcal{M}=\epsilon R T \Gamma^{*} / \mu_{1} U$ & Marangoni number & 0.38 \\
$\mathcal{P}=D_{s} / U L_{o p}$ & Péclet number & $5.9 \cdot 10^{-5}$ \\
$\Upsilon=\epsilon^{2} \mu_{2} / \mu_{1}$ & Viscosities ratio & $4.8 \cdot 10^{-2}$ \\
$\mathcal{E}=E_{0} / \epsilon U \nu$ & Evaporation mass transfer coefficient & $9.2 \cdot 10^{-5}$ \\
$\hat{\nu}=\nu / \delta \epsilon L_{o p}$ & Evaporation constant & $3.3 \cdot 10^{-2}$ \\
$\mathcal{T}=\gamma_{2 a i r} / \gamma_{12}$ & Surface tension ratio & 0.4 \\
$\varrho=\rho_{2} / \rho_{1}$ & Density ratio & 0.9 \\
\hline
\end{tabular}

TABLE 2. Dimensionless parameters and their typical values.

- To preserve surface tension effects, we suppose that the reduced capillary numbers $\mathcal{C}_{1}=\epsilon^{3} / C a_{1}$ and $\mathcal{C}_{2}=\epsilon / C a_{2}$ are $O(1)$.

- To ensure that the pressure drives a flow in both layers, we suppose that $\Upsilon=\epsilon^{2} \mu^{*}$ is $O(1)$.

- To keep gravitational forces in the model, we suppose that $\mathcal{S}_{1}=\epsilon^{2} S t_{1}$ and $\mathcal{S}_{2}=S t_{2}$ are both $O(1)$.

- Marangoni forces are required at the water-lipid interface, and so we suppose that $\mathcal{M}=\epsilon M a$ is $O(1)$.

- To keep diffusion in the model despite the tiny inverse Péclet number, we assume (for now) that it is $O(1)$.

- While $\delta$ is small, we will treat it as $O(1)$.

- We will take various sublimits of the model that we derive using these assumptions in section 5 .

There are (of course) other parameter choices that would result in other distinguished limits of the model, but we do not consider them in this paper.

\subsection{Reduction to thin film limit}

We seek perturbation expansions for all the dependent variables of the form $f \sim f^{(0)}+$ $\epsilon^{2} f^{(1)}+\cdots$. With the assumptions given in section 3.3 , the leading-order equations 
(dropping superscripts for the leading-order terms) become

$$
\begin{aligned}
p_{1 x} & =u_{1 y y}-\mathcal{S}_{1}, \\
p_{1 y} & =0, \\
u_{1 x}+v_{1 y} & =0,
\end{aligned}
$$

for the aqueous layer, while for the non-polar lipid layer we have

$$
\begin{aligned}
u_{2 \zeta \zeta} & =0, \\
\delta p_{2 \zeta} & =v_{2 \zeta \zeta}, \\
\delta u_{2 x}-h_{1 x} u_{2 \zeta}+v_{2 \zeta} & =0,
\end{aligned}
$$

and for the polar lipid we have

$$
\Gamma_{t}+\left[u_{1}\left(x, h_{1}\right) \Gamma\right]_{x}=\mathcal{P} \Gamma_{x x} .
$$

The leading-order boundary conditions are as follows. At $y=0$ :

$$
u_{1}=v_{1}=0 .
$$

At $y=h_{1}($ or $\zeta=0)$ :

$$
\begin{aligned}
\mathbf{u}_{1} & =\mathbf{u}_{2}, \\
v_{1}-h_{1 t}-h_{1 x} u_{1} & =\mathcal{E} E \\
\Upsilon p_{2}-p_{1}-\frac{2 \Upsilon}{\delta}\left(v_{2 \zeta}-h_{1 x} u_{2 \zeta}\right) & =\mathcal{C}_{1} h_{1 x x}, \\
u_{2 \zeta} & =0,
\end{aligned}
$$

At $y=h\left(\right.$ or $\left.\zeta=h_{2}\right)$ :

$$
\begin{aligned}
v_{2}-h_{t}-h_{x} u_{2} & =\mathcal{E} E \\
-\delta p_{2}+2\left(v_{2 \zeta}-h_{x} u_{2 \zeta}\right) & =\delta \mathcal{C}_{2} h_{x x}, \\
u_{2 \zeta} & =0,
\end{aligned}
$$

We can easily see from $(3.18 a),(3.20 e)$ and $(3.20 h)$ that the leading-order solution for $u_{2}$ is

$$
u_{2}=u_{2}(x, t),
$$

i.e. the flow in the non-polar lipid layer is extensional and, as it is often the case for liquid flow between two free surfaces, in order to close the leading-order model we need to use some of the field equations and boundary conditions at the next order. The relevant formulae are the $O\left(\epsilon^{2}\right)$ versions of $(3.7 b)-(3.7 c)$, namely

$$
\begin{aligned}
-u_{2 \zeta \zeta}^{(1)}+\delta\left(\delta p_{2 x}-h_{1 x} p_{2 \zeta}\right) & =\delta^{2} u_{2 x x}-2 \delta h_{1 x} u_{2 x \zeta}-\delta h_{1 x x} u_{2 \zeta}+h_{1 x}^{2} u_{2 \zeta \zeta}-\delta^{2} \mathcal{S}_{2}, \\
\delta p_{2 \zeta}^{(1)}-v_{2 \zeta \zeta}^{(1)} & =\delta^{2} v_{2 x x}-2 \delta h_{1 x} v_{2 x \zeta}-\delta h_{1 x x} v_{2 \zeta}+h_{1 x}^{2} v_{2 \zeta \zeta}
\end{aligned}
$$

the $O\left(\epsilon^{2}\right)$ version of the shear stress condition $(3.10 c)$ at $y=h_{1}$ :

$$
u_{1 y}-\frac{\Upsilon}{\delta} G(x)=-\mathcal{M} \Gamma_{x},
$$

where

$$
G(x)=\left(u_{2 \zeta}^{(1)}+h_{1 x} v_{2 \zeta}-2 \delta h_{1 x} u_{2 x}+\delta v_{2 x}\right)_{y=h_{1}},
$$


and the $O\left(\epsilon^{2}\right)$ version of the shear stress condition (3.11c) at $y=h$ :

$$
u_{2 \zeta}^{(1)}+2 h_{x}\left(v_{2 \zeta}-\delta u_{2 x}+h_{1 x} u_{2 \zeta}\right)-h_{x}^{2} u_{2 \zeta}+\delta v_{2 x}-h_{1 x} v_{2 \zeta}=0 .
$$

We start by noting that, from $(3.17 b), p_{1}=p_{1}(x)$ is independent of $y$. Then, using $(3.20 d)$, we obtain

$$
p_{1}(x)=\Upsilon\left[p_{2}-\frac{2}{\delta}\left(v_{2 \zeta}-h_{1 x} u_{2 \zeta}\right)\right]_{y=h_{1}}-\mathcal{C}_{1} h_{1 x x} .
$$

Introducing

$$
F(x)=\left(p_{2}-\frac{2}{\delta} v_{2 \zeta}\right)_{y=h_{1}},
$$

integrating (3.17a) and imposing the conditions (3.23) and (3.20a), we find that

$$
u_{1}(x, y)=\left[\Upsilon F^{\prime}(x)-\mathcal{C}_{1} h_{1 x x x}+\mathcal{S}_{1}\right]\left(\frac{y^{2}}{2}-h_{1} y\right)+\left[\frac{\Upsilon}{\delta} G(x)-\mathcal{M} \Gamma_{x}\right] y .
$$

Integrating the continuity equation $(3.17 c)$ and using $(3.20 a)$ and $(3.20 c)$, gives the lubrication equation for the aqueous layer

$$
h_{1 t}+\left(h_{1} \bar{u}_{1}\right)_{x}=-\mathcal{E} E,
$$

where

$$
\bar{u}_{1}=-\left[\Upsilon F^{\prime}(x)-\mathcal{C}_{1} h_{1 x x x}+\mathcal{S}_{1}\right] \frac{h_{1}^{2}}{3}+\left[\frac{\Upsilon}{\delta} G(x)-\mathcal{M} \Gamma_{x}\right] \frac{h_{1}}{2},
$$

is the cross-sectionally averaged horizontal velocity. We note that both $F$ and $G$ depend on the solution in the upper lipid layer (see (3.24) and (3.27)).

In the non-polar lipid layer we find, from (3.18c) and (3.20b), that the vertical velocity is given by

$$
v_{2}(x, \zeta)=-\delta u_{2 x}(x) \zeta+v_{1}\left(x, h_{1}\right)
$$

Using the kinematic condition $(3.20 f)$, we easily arrive at the conservation of mass equation for the non-polar lipid layer

$$
h_{2 t}+\left(h_{2} u_{2}\right)_{x}=0 .
$$

From $(3.18 b)$ and $(3.20 \mathrm{~g})$, we find that the pressure in the lipid layer is given by

$$
p_{2}(x)=-2 u_{2 x}-\mathcal{C}_{2} h_{x x} .
$$

The solution in the non-polar lipid layer allows us to simplify our expression for $F(x)$, since we find that

$$
F^{\prime}(x)=\left(p_{2 x}-\frac{2}{\delta} v_{2 \zeta x}\right)_{y=h_{1}}=p_{2 x}+2 u_{2 x x}=-\mathcal{C}_{2} h_{x x x} .
$$

In order to solve for $u_{2}$, we need to consider the order $O\left(\epsilon^{2}\right)$ equations and boundary conditions given in $(3.22 a),(3.22 b),(3.23)$ and (3.25). After slight manipulation, (3.22a) reads

$$
u_{2 \zeta \zeta}^{(1)}=-\delta^{2}\left(3 u_{2 x x}+\mathcal{C}_{2} h_{x x x}-\mathcal{S}_{2}\right) .
$$

Integrating (3.35) with respect to $\zeta$, imposing (3.25) and using the continuity of velocity at $y=h_{1}, u_{2}(x, t)=u_{1}\left(x, h_{1}, t\right)$, we find that $u_{2}$ satisfies

$$
4 \delta \Upsilon\left(h_{2} u_{2 x}\right)_{x}-\frac{u_{2}}{h_{1}}=\frac{h_{1}}{2}\left(\mathcal{S}_{1}-\mathcal{C}_{1} h_{1 x x x}-\Upsilon \mathcal{C}_{2} h_{x x x}\right)+\delta \Upsilon h_{2}\left(\mathcal{S}_{2}-\mathcal{C}_{2} h_{x x x}\right)+\mathcal{M} \Gamma_{x} .
$$


The expressions for $u_{1}$ and $\bar{u}_{1}$ can be thus be simplified to

$$
\begin{aligned}
& u_{1}=\frac{1}{2}\left(\mathcal{C}_{1} h_{1 x x x}+\Upsilon \mathcal{C}_{2} h_{x x x}-\mathcal{S}_{1}\right)\left(h_{1} y-y^{2}\right)+\frac{u_{2}}{h_{1}} y, \\
& \bar{u}_{1}=\frac{h_{1}^{2}}{12}\left(\mathcal{C}_{1} h_{1 x x x}+\Upsilon \mathcal{C}_{2} h_{x x x}-\mathcal{S}_{1}\right)+\frac{u_{2}}{2} .
\end{aligned}
$$

Finally, given that $u_{1}\left(x, h_{1}, t\right)=u_{2}(x, t)$, the polar lipid equation (3.19) reads

$$
\Gamma_{t}+\left(u_{2} \Gamma-\mathcal{P} \Gamma_{x}\right)_{x}=0 .
$$

\subsection{Summary}

Our model thus comprises four coupled equations for the four unknowns $h_{1}, h_{2}, u_{2}$ and $\Gamma$,

$$
\begin{aligned}
h_{1 t}+\left(h_{1} \bar{u}_{1}\right)_{x} & =-\mathcal{E} \frac{\hat{\nu}}{\hat{\nu}+h_{2}}, \\
h_{2 t}+\left(h_{2} u_{2}\right)_{x} & =0 \\
\Gamma_{t}+\left(\Gamma u_{2}-\mathcal{P} \Gamma_{x}\right)_{x} & =0 \\
4 \delta \Upsilon\left(h_{2} u_{2 x}\right)_{x} & =\frac{1}{h_{1}}\left(4 u_{2}-6 \bar{u}_{1}\right)+\delta h_{2}\left[\varrho \mathcal{S}_{1}-\mathcal{T} \mathcal{C}_{1}\left(h_{1 x x x}+\delta h_{2 x x x}\right)\right]+\mathcal{M} \Gamma_{x},
\end{aligned}
$$

where

$$
\bar{u}_{1}=\frac{h_{1}^{2}}{12}\left[(1+\mathcal{T}) \mathcal{C}_{1} h_{1 x x x}+\delta \mathcal{T} \mathcal{C}_{1} h_{2 x x x}-\mathcal{S}_{1}\right]+\frac{u_{2}}{2},
$$

which must be solved together with the boundary conditions (3.13), (3.14), and the initial conditions in (3.16). Remembering that the leading-order fluxes are $q_{1}=h_{1} \bar{u}_{1}, q_{2}=h_{2} u_{2}$ and $q_{s}=\Gamma u_{2}-\mathcal{P} \Gamma_{x}$, the boundary conditions can be written as

$$
\begin{aligned}
& h_{1}=1, \quad h_{2 x}=0, \quad \bar{u}_{1}=0, \quad u_{2}=0, \quad \Gamma u_{2}-\mathcal{P} \Gamma_{x},=0 \quad \text { at } x=0, \\
& h_{1}=1, \quad h_{2 x}=0, \quad \bar{u}_{1}=\chi \dot{L}, \quad u_{2}=\dot{L}, \quad \Gamma u_{2}-\mathcal{P} \Gamma_{x}=\Gamma \dot{L}, \quad \text { at } x=L,
\end{aligned}
$$

and the initial conditions are

$$
\Gamma=1, \quad h_{2}=1, \quad h_{1}=\frac{3 \hat{V}}{2}-\frac{1}{2}+\frac{3}{2}(1-\hat{V})\left(\frac{2 x}{\lambda}-1\right)^{2}, \quad \text { at } t=0 .
$$

We have introduced new parameters $\mathcal{T}$ and $\varrho$, defined via

$$
\Upsilon \mathcal{C}_{2}=\frac{\gamma_{2 a i r}}{\gamma_{12}} \mathcal{C}_{1}=\mathcal{T} \mathcal{C}_{1} \quad \text { and } \quad \Upsilon \mathcal{S}_{2}=\frac{\rho_{2}}{\rho_{1}} \mathcal{S}_{1}=\varrho \mathcal{S}_{1},
$$

to make it clear that neither $\Upsilon \mathcal{C}_{2}$ nor $\Upsilon \mathcal{S}_{2}$ actually vary as the viscosity of the upper layer varies.

\section{Results}

In this section we examine behaviour of the model presented in (3.39) as we change some of the key parameters. We note that there is no time derivative in $(3.39 d)$, and thus the system (3.39) is a partial differential algebraic system. In order to solve this system, we map the problem (3.39) onto a fixed computational domain (Jones et al. 2006), code in $\mathrm{C}++$, and solve using a second-order-accurate finite-difference scheme along with a backward Euler time-stepping method, where the time step is chosen based on an error 
bound. A Newton-Raphson method is used to solve the resulting system of nonlinear equations. This numerical method is used in all the simulations in this paper except for the paradigm problem in appendix $\mathrm{C}$. We have taken special care to use a consistent initialisation of the system. A convergence study is shown in appendix B and based on this, we have used 10000 spatial grid points in the results presented below.

\subsection{Blink}

First we show the evolution of the film thickness, the concentration of polar lipid, the concentration of the non-polar lipid and the velocity of the non-polar lipid during the blink. We use the parameters given in table 2, and we show the numerical solution in figure 3 .

The general evolution of the thickness of the aqueous layer and the concentration of polar lipid as seen in figures 3(a) and 3(b) are almost identical to that presented in Aydemir et al. (2011). The behaviour is described in detail in there. Here we simply note that, after the blink, the polar lipid is mainly present in two reservoirs, one near each eyelid. The non-polar lipid distributes itself similarly into two reservoirs, but there is a thin "precursor" layer that spreads up the upper eyelid surface, as seen in figure 3(c) and the close up in figure 3(d). We note that this layer is well resolved; there are 500 grid points in figure 3(d). In the central region between the two reservoirs, there is a reduced film of non-polar lipid and thus during the interblink, we will expect there to be much more evaporation in this region compared with near the lids.

\subsection{Interblink}

Figure 4 shows the evolution during the interblink. As during the blink, the thickness of the aqueous layer and the concentration of polar lipid have similar dynamics to those shown in Aydemir et al. (2011). The dynamics of the non-polar lipid layer are more interesting as can be seen in figures 4(c) and 4(d). Near the lower eyelid, a ridge forms which travels upwards over the central tear film region and tracking its motion reflects the spread of the non-polar lipid layer over the surface, as seen in the pictures in Yokoi et al. (2008).

Near the upper eyelid, the precursor film retracts and a second ridge forms at the edge of the meniscus, located at the upper stagnation point of the flow in the non-polar lipid layer. The non-polar lipid layer is thicker at this upper ridge than at the lower ridge, but the overall film thickness $\left(h=h_{1}+\delta h_{2}\right)$ there is ten times smaller than the total thickness at the lower ridge. We plot $h$ in figure 5 and find that the ridge in $h_{2}$ is indistinguishable. We will discuss the ridge further in section 4.3.3.

\subsection{Dependence on parameter groups}

In this subsection we consider variations of the parameters to understand the effect that different parameters have on the behaviour of the system. Specifically, we look at changes in the evaporation rate $\mathcal{E}$, the Marangoni number $\mathcal{M}$ and the Péclet number $\mathcal{P}$, respectively.

\subsubsection{Effect of evaporation on the tear film thickness}

A key outcome of having a non-uniform layer of non-polar lipid is that the rate of evaporation will vary across the tear film surface. In figure 6 we examine the aqueous layer thickness at the end of the interblink $\left(t=T_{f}=\Delta \hat{t}_{u}+\Delta \hat{t}_{i}=26.5\right)$ for four different evaporation rates: $\mathcal{E}=0, \mathcal{E}=0.5 \mathcal{E}^{*}, \mathcal{E}=\mathcal{E}^{*}$ and $\mathcal{E}=1.5 \mathcal{E}^{*}$, where $\mathcal{E}^{*}$ is the "standard" value of $\mathcal{E}$ given in table 2 . We note that all these values are physically realisable across the spectrum of normal and dry eye patients. We use $h_{1}(x ; \mathcal{E})$ to denote the final thickness for 

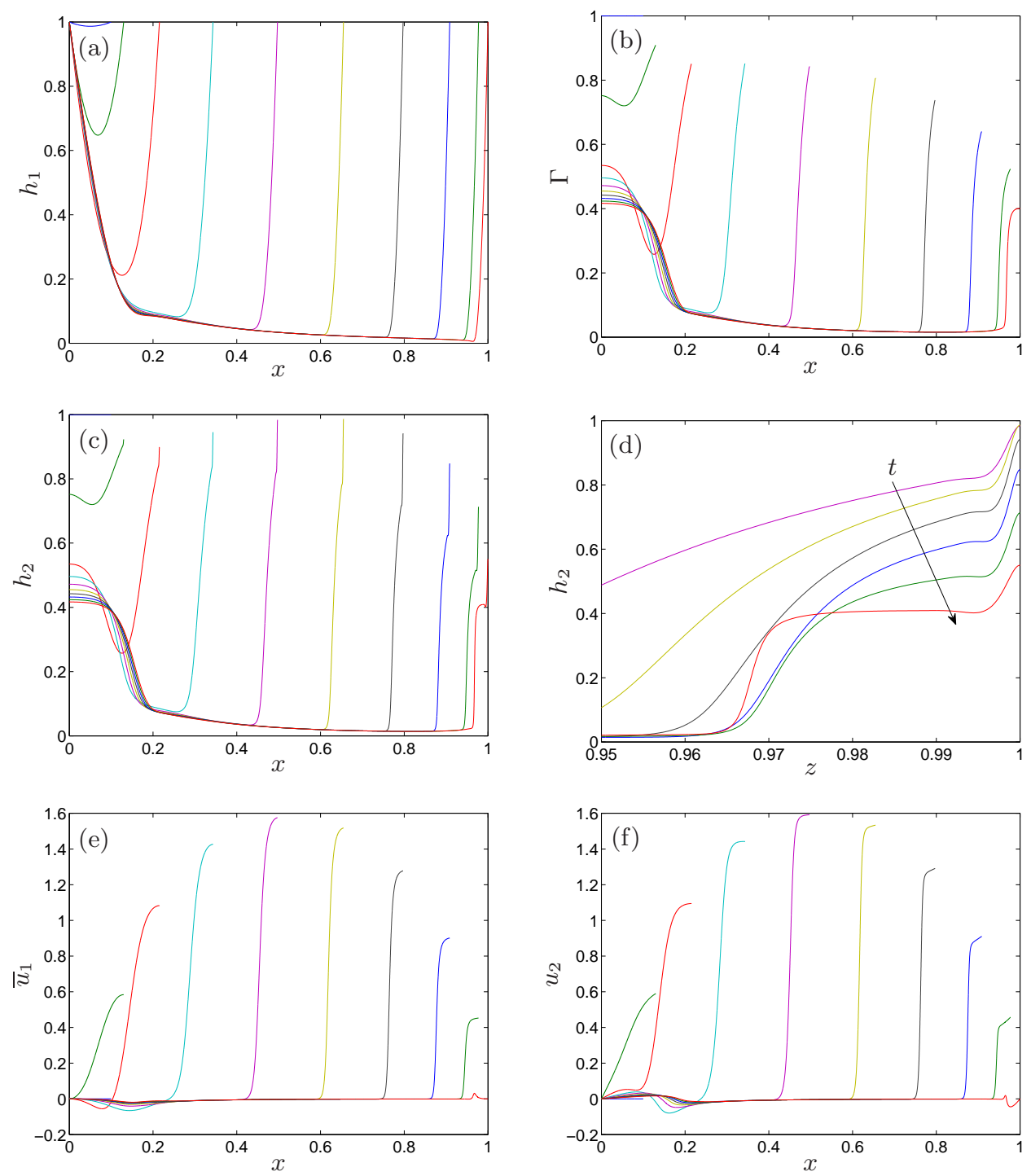

FigURE 3. Numerical solution of (3.39) showing the evolution of $h_{1}, \Gamma, h_{2}, \bar{u}_{1}$ and $u_{2}$ during the blink, for $t=0,0.1, \ldots, 0.9$. (d) Behaviour of $h_{2}$ close to the upper eyelid $(z=x / L(t))$ for $t=0.4,0.5, \ldots, 0.9$. The values of the parameters are given in table 2 .

a given evaporation rate $\mathcal{E}$. In figure $6(\mathrm{a})$ we plot the evolution of the minimum aqueous layer thickness for the four evaporation rates.

We find that the minimum tear film thickness has changed by $58 \%$ due to evaporation by the end of the interblink. Figure 6(b) shows the relative difference between the solution with various nonzero evaporation rates and the solution with evaporation set to zero, $h_{1}(x ; 0)$. We see that evaporation has a significant effect on the local film thickness near the upper black line by the end on the interblink, but there is no observable change in the menisci. As discussed in Zubkov et al. (2012), evaporative reduction of the aqueous layer can result in significant increases in osmolarity (see Liu et al. 2009), and thus discomfort to the patient. We note that our simulations show that the film thins to a thickness where 

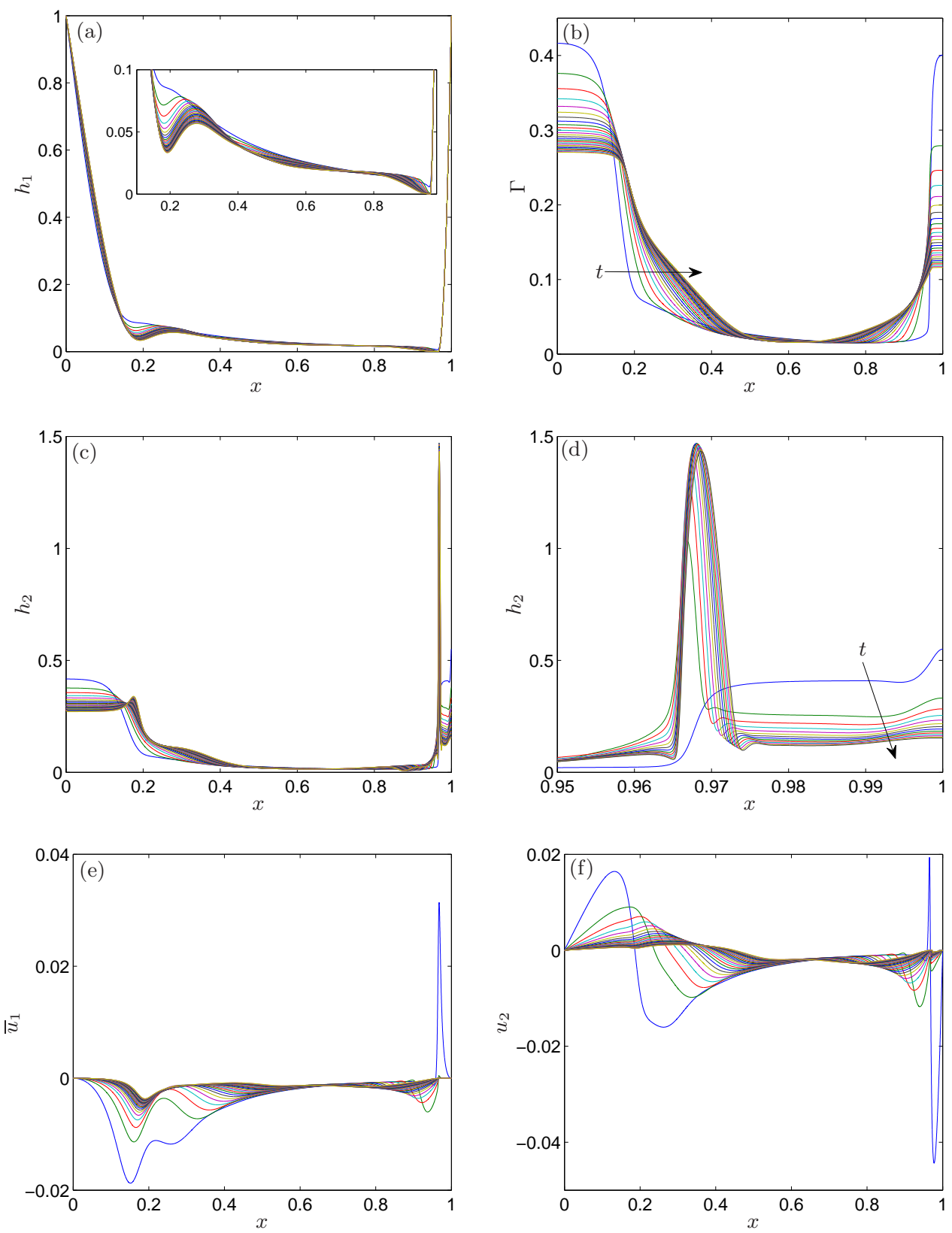

FiguRE 4. Numerical solution of (3.39) showing the evolution of $h_{1}, \Gamma, h_{2}, \bar{u}_{1}$ and $u_{2}$ during the interblink, for $t=0.9,1.9, \ldots, 26.5$. (d) Behaviour of $h_{2}$ close to the upper eyelid for $t=0.9,2.9,4.9, \ldots, 26.5$. The values of the parameters are given in table 2 .

intermolecular forces might become important; we do not examine this further in this paper.

\subsubsection{Effect of surface tension variations on tear film evolution}

We alter the Marangoni number to assess how the strength of the surface tension variations affect tear film dynamics. We find that increasing $\mathcal{M}$ has a marked effect on 

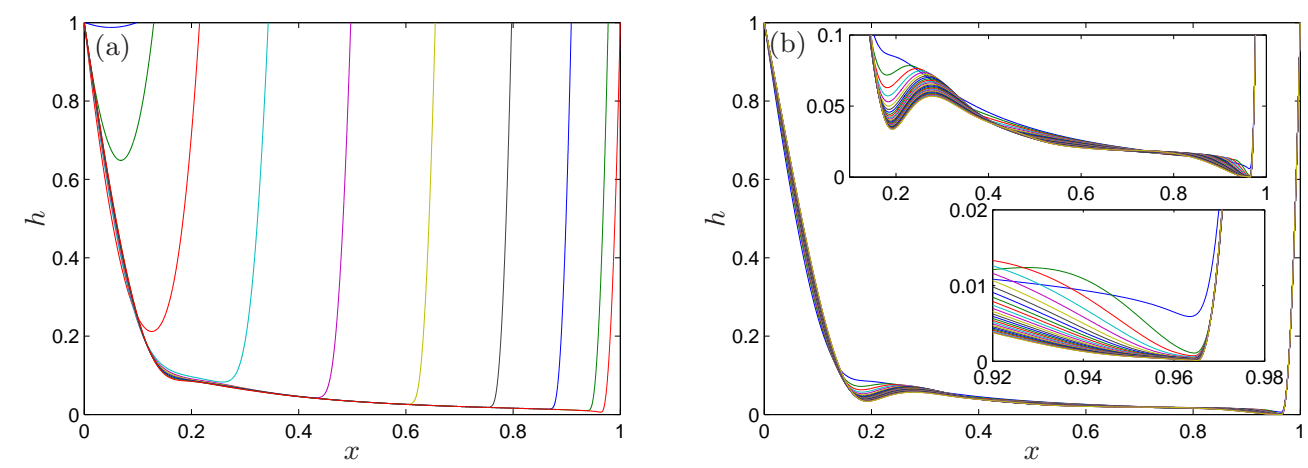

FiguRE 5. Numerical solution of (3.39) showing the overall tear film thickness $h=h_{1}+\delta h_{2}$. (a) upstroke a times $t=0,0.1, \ldots, 0.9$. (b) interblink at times $t=0.9,1.9, \ldots, 26.5$. The values of the parameters are given in table 2 .
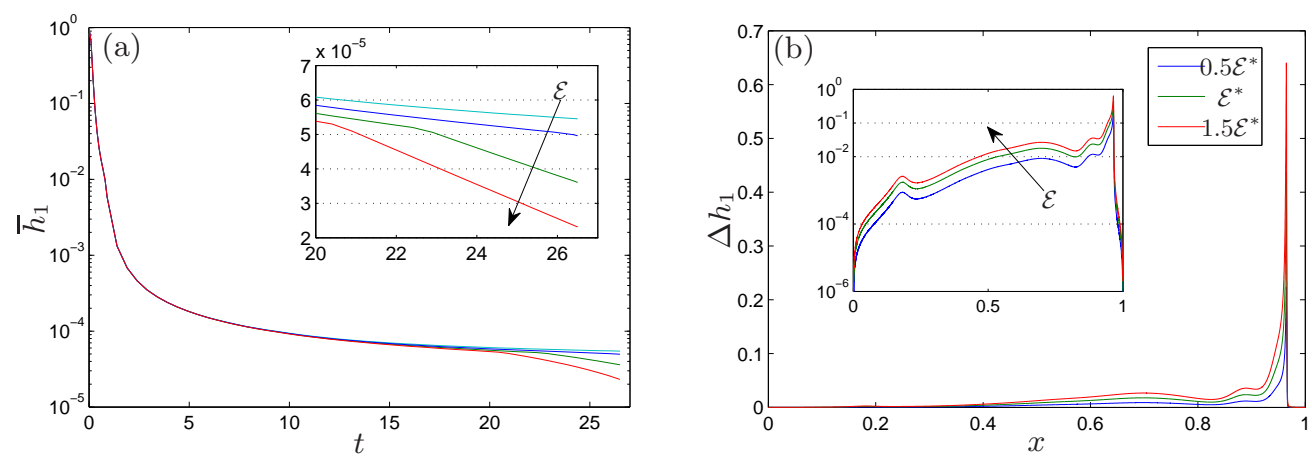

FiguRE 6. Effect of changing the evaporation constant $\mathcal{E}$ on the aqueous layer thickness $h_{1}$ from (3.39), with $\mathcal{E}=0,0.5 \mathcal{E}^{*}, \mathcal{E}^{*}$ and $1.5 \mathcal{E}^{*}$. (a) Evolution in time of the minimum value of $h_{1}, \bar{h}_{1}:=\min _{x} h_{1}(x, t)$, as the evaporation rate increases from 0 to $1.5 \mathcal{E}^{*}$ as shown with the arrow. Inset shows the behaviour for late time. (b) Relative difference in $h_{1}$ at $t=T_{f}$ (end of the interblink) between the solutions with positive evaporation $\left(\mathcal{E}=0.5 \mathcal{E}^{*}, \mathcal{E}^{*}\right.$ and $\left.1.5 \mathcal{E}^{*}\right)$ and the solution with $\mathcal{E}=0$. We use $\Delta h_{1}(x)=\left[h_{1}(x ; \mathcal{E})-h_{1}(x ; 0)\right] / h_{1}(x ; 0)$. Inset shows $\log$ of $\Delta h_{1}$. The values of all parameters including the default value of $\mathcal{E}, \mathcal{E}^{*}$, are given in table 2 .

all the dependent variables. In figure 7 we see that increasing $\mathcal{M}$ causes an increase in the thickness of the aqueous layer across the tear film central region and it thickens the minimum in the aqueous layer thickness. Increasing $\mathcal{M}$ also causes both the polar lipid and non-polar lipid to spread more quickly across the eye surface and to become more uniform (we show the evolution of $h_{2}$ for three different $\mathcal{M}$ values in figure 8 ). We note that the position of the ridge in the non-polar lipid layer near the lower eyelid is located in the vicinity of the black line near the lower eyelid, and that increasing the Marangoni number suppresses the ridge that forms at the upper meniscus.

\subsubsection{Effect of diffusion of polar lipid on tear film evolution}

Figures 9 and 10 show the changes in the polar and non-polar lipid layers respectively, as we change the Péclet number $\mathcal{P}$ from its value in table 2 , which we denote by $\mathcal{P}^{*}$. We see in figure 9 that the distribution of polar lipid in the cases $\mathcal{P}=0, \mathcal{P}^{*}$ and $2 \mathcal{P}^{*}$ are identical except right by the upper eyelid where the concentration changes by around 10 

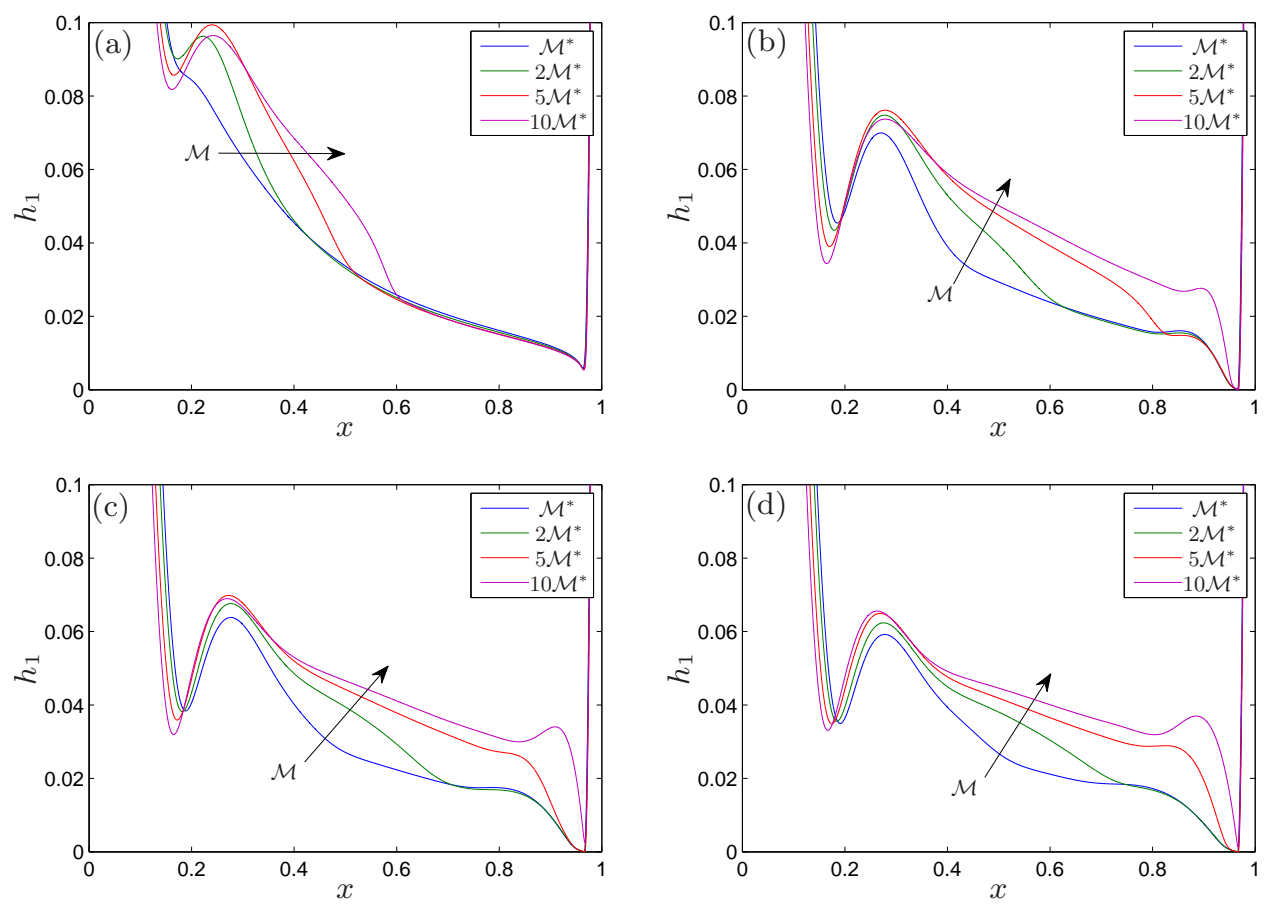

FiguRE 7 . Effect of changing $\mathcal{M}$ on the aqueous layer thickness $h_{1}$ during the interblink from (3.39) at times $t=0.9$ in (a), $t=7.9$ in (b), $t=14.9$ in (c), and $t=21.9$ in (d). The values of all parameters including the default value of $\mathcal{M}, \mathcal{M}^{*}$, are given in table 2 .

$\%$ between the $\mathcal{P}=0$ and $2 \mathcal{P}^{*}$ cases. We note that we can vary $\mathcal{P}$ by altering the speed of the blink.

In contrast, we see in figure 10 that there is a marked change in the behaviour of the non-polar lipid as the Péclet number is increased. We see in figure 10(a,c,e) that increasing the Péclet number creates a precursor layer in the non-polar lipid near the upper eyelid and that this layer intensifies as the eye opens. During the interblink, we see in figure $10(\mathrm{~b}, \mathrm{~d}, \mathrm{f})$ that increasing the Péclet number intensifies the ridges both at the lower and upper eyelids. This may be counterintuitive, since one may think that increasing $\mathcal{P}$ increases the diffusion strength and therefore the solution should smooth out.

It is clear from figure 10 that the formation of the ridge in $h_{2}$ near the upper eyelid is affected by the magnitude of the Péclet number since for $\mathcal{P}=0$ the ridge does not form (see figures 10(a) and 10(b)). In this case, the solutions for $h_{2}$ and $\Gamma$ are in fact identical. What is somewhat surprising is that adding diffusion in the polar lipid equation has a "destabilizing" effect on the non-polar lipid rather than the more expected diffusive smoothing. However, the reader should remember that this is a coupled system and that the surface velocity $u_{2}$, which appears both in the polar and non-polar flux terms, is obtained by solving the $(3.39 d)$.

In order to understand what happens to $u_{2}$ when $\mathcal{P} \neq 0$, it is useful to compare the effective velocity of $\Gamma$ in $(3.39 c), u_{\Gamma}=u_{2}-\mathcal{P} \Gamma_{x} / \Gamma$, with $u_{2}$. In figure 11 we plot the evolution during the interblink of both types of lipid and their respective velocities in the area near the upper eyelid where the ridge forms. While the polar lipid velocity $u_{\Gamma}$ is negative and monotonic for all times (see figure 11(c)) and nothing interesting occurs 

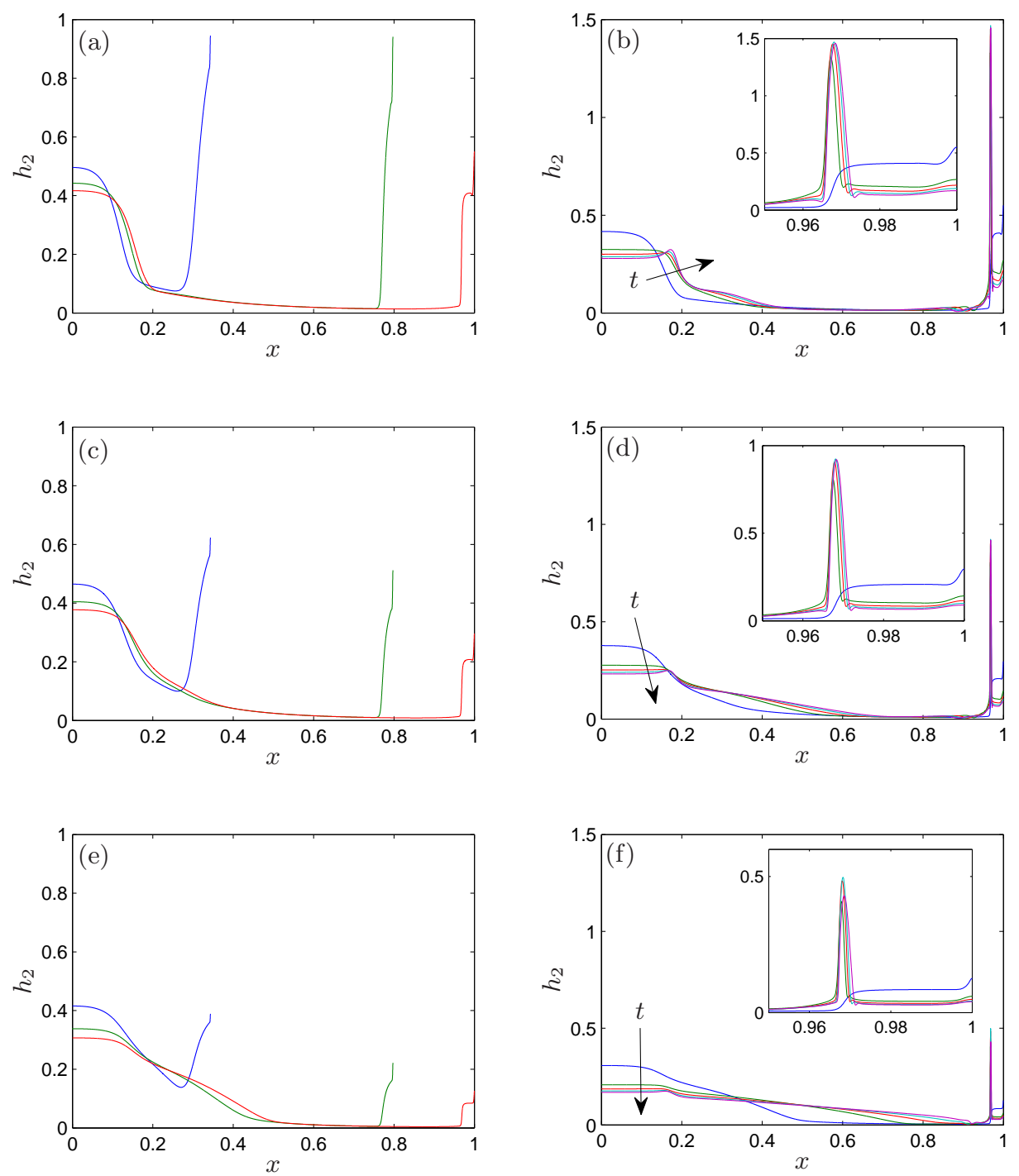

FiguRE 8. Effect of changing $\mathcal{M}$ on the nonpolar lipid thickness $h_{2}$ from (3.39), where values used are $\mathcal{M}^{*}$ in $(\mathrm{a}, \mathrm{b}), 2 \mathcal{M}^{*}$ in $(\mathrm{c}, \mathrm{d})$, and $5 \mathcal{M}^{*}$ in $(\mathrm{e}, \mathrm{f})$. (a,c,e) Upstroke at times $t=0,0.1, \ldots, 0.9$; (b,d,f) interblink at times $t=0.9,1.9, \ldots, 26.5$."

in the polar lipid concentration (figure 11(a)), the non-polar lipid velocity $u_{2}$ has a nontrivial shape in this region, as we can see in figure 11(d). For times $t>14.40$, we see in the inset of figure 11(d) that two new stagnation points appear in $u_{2}$ (from the ones already present closer to the lower eyelid, see figure 4(f)), but this is not the reason why the non-polar ridge appears as it is already well formed for much earlier times (see figure 11(b)). Instead, the changes in convexity of $u_{2}$ seem to correlate with the ridge formation, since the ridge 'bases' or 'contact lines' appear to move with the points of the change of convexity in $u_{2}$. 

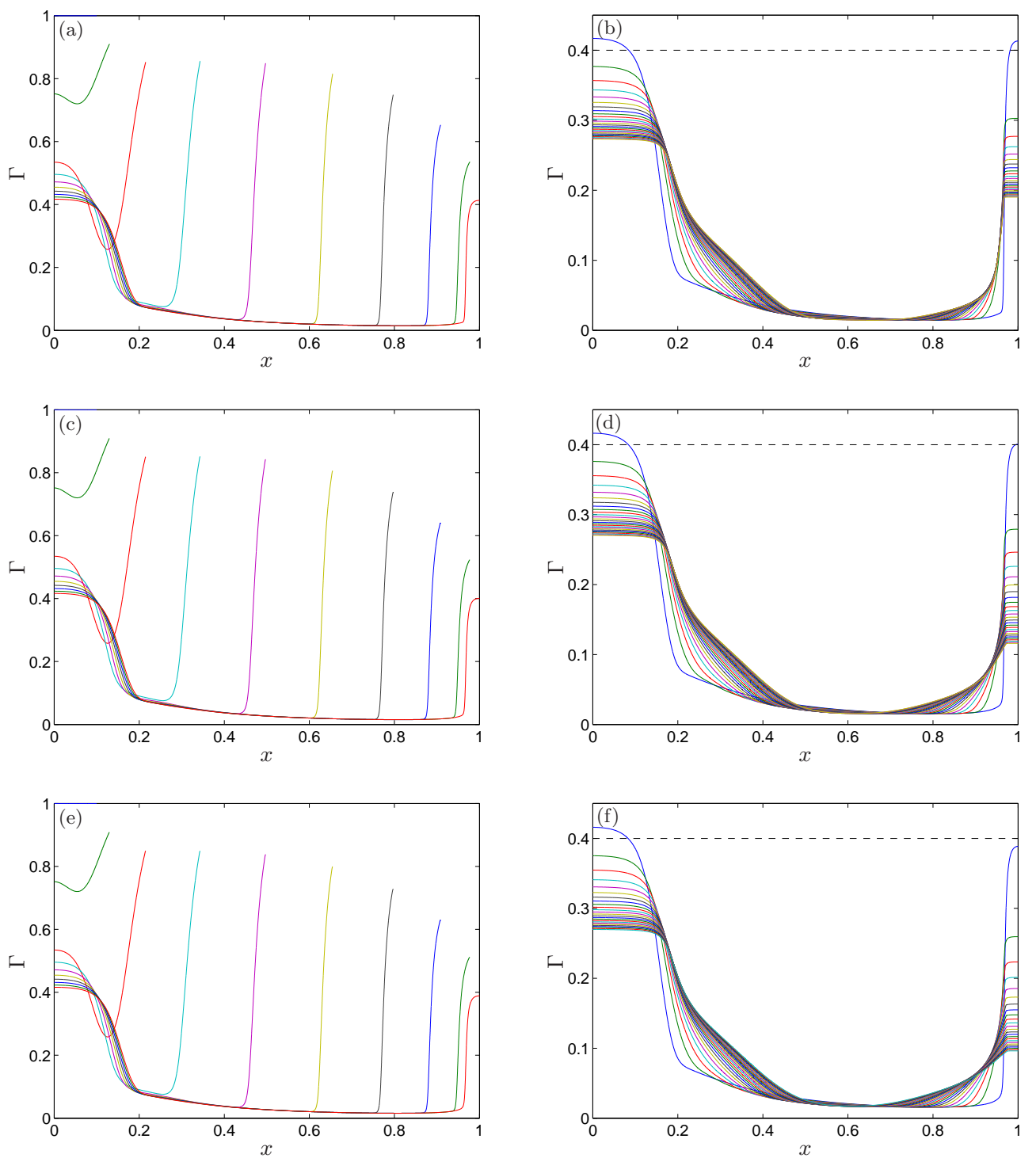

Figure 9. Effect of changing $\mathcal{P}$ on the polar lipid concentration $\Gamma$ from (3.39), where values used are $\mathcal{P}=0$ in $(\mathrm{a}, \mathrm{b}), \mathcal{P}=\mathcal{P}^{*}$ in $(\mathrm{c}, \mathrm{d})$, and $\mathcal{P}=2 \mathcal{P}^{*}$ in $(\mathrm{e}, \mathrm{f})$. (a,c,e) Upstroke at times $t=0,0.1, \ldots, 0.9 ;(\mathrm{b}, \mathrm{d}, \mathrm{f})$ interblink at times $t=0.9,1.9, \ldots, 26.5$. The values of all parameters including the default value of $\mathcal{P}, \mathcal{P}^{*}$, are given in table 2 .

Therefore, the zoomed graphs in figure 11 seem to indicate that, when the polar lipid velocity is modified by $\mathcal{P}, u_{2}$ is the one to readjust. The new non-polar lipid velocity (which may be useful to think of as $u_{\Gamma}+\mathcal{P} \Gamma_{x} / \Gamma$ ) turns out to have a complicated shape near the upper eyelid. The location of this double change in convexity is given by the stagnation point in the aqueous layer (see figure 12). 

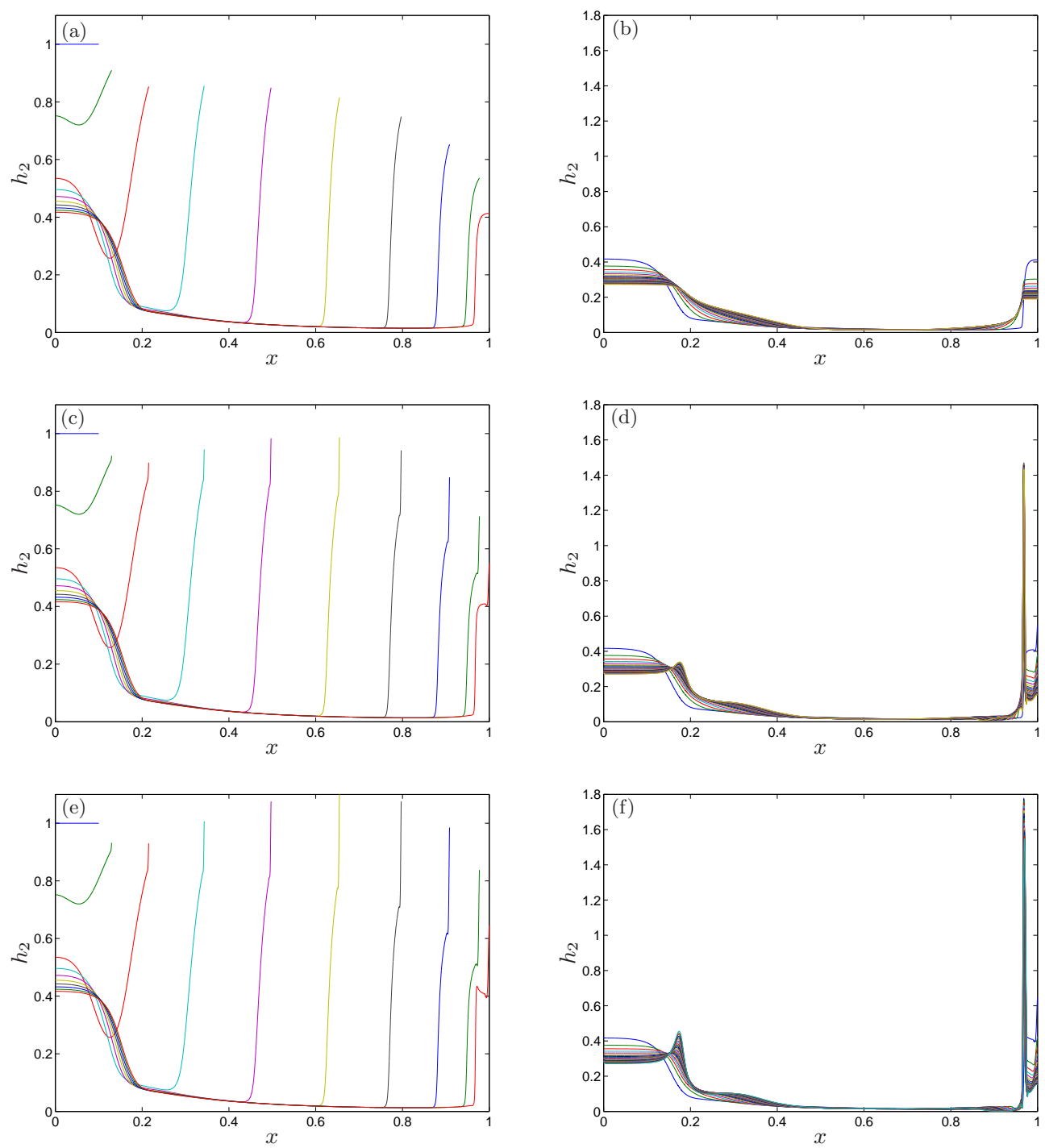

FiguRE 10. Effect of changing $\mathcal{P}$ on the polar lipid thickness $h_{2}$ from (3.39), where values used are $\mathcal{P}=0$ in $(\mathrm{a}, \mathrm{b}), \mathcal{P}=\mathcal{P}^{*}$ in $(\mathrm{c}, \mathrm{d})$, and $\mathcal{P}=2 \mathcal{P}^{*}$ in $(\mathrm{e}, \mathrm{f})$. (a,c,e) Upstroke at times $t=0,0.1, \ldots, 0.9 ;(\mathrm{b}, \mathrm{d}, \mathrm{f})$ interblink at times $t=0.9,1.9, \ldots, 26.5$.

\section{Reduced models}

In this section we reduce the distinguished parameter limit (3.39) to several submodels. In each case, we also discuss the appropriate the boundary conditions to be applied.

5.1. Non-polar lipid layer has no effect of tear film, $\mathcal{P} \ll 1, \delta \ll 1, \mathcal{T} \ll 1$ and $\mathcal{E} \ll 1$

In this limit, neither the surface diffusivity of the polar lipid, nor the viscosity of the non-polar lipid layer, nor the surface tension of the non-polar lipid layer/air interface, nor evaporation are strong enough to affect the fluid flow in the aqueous layer. We find 

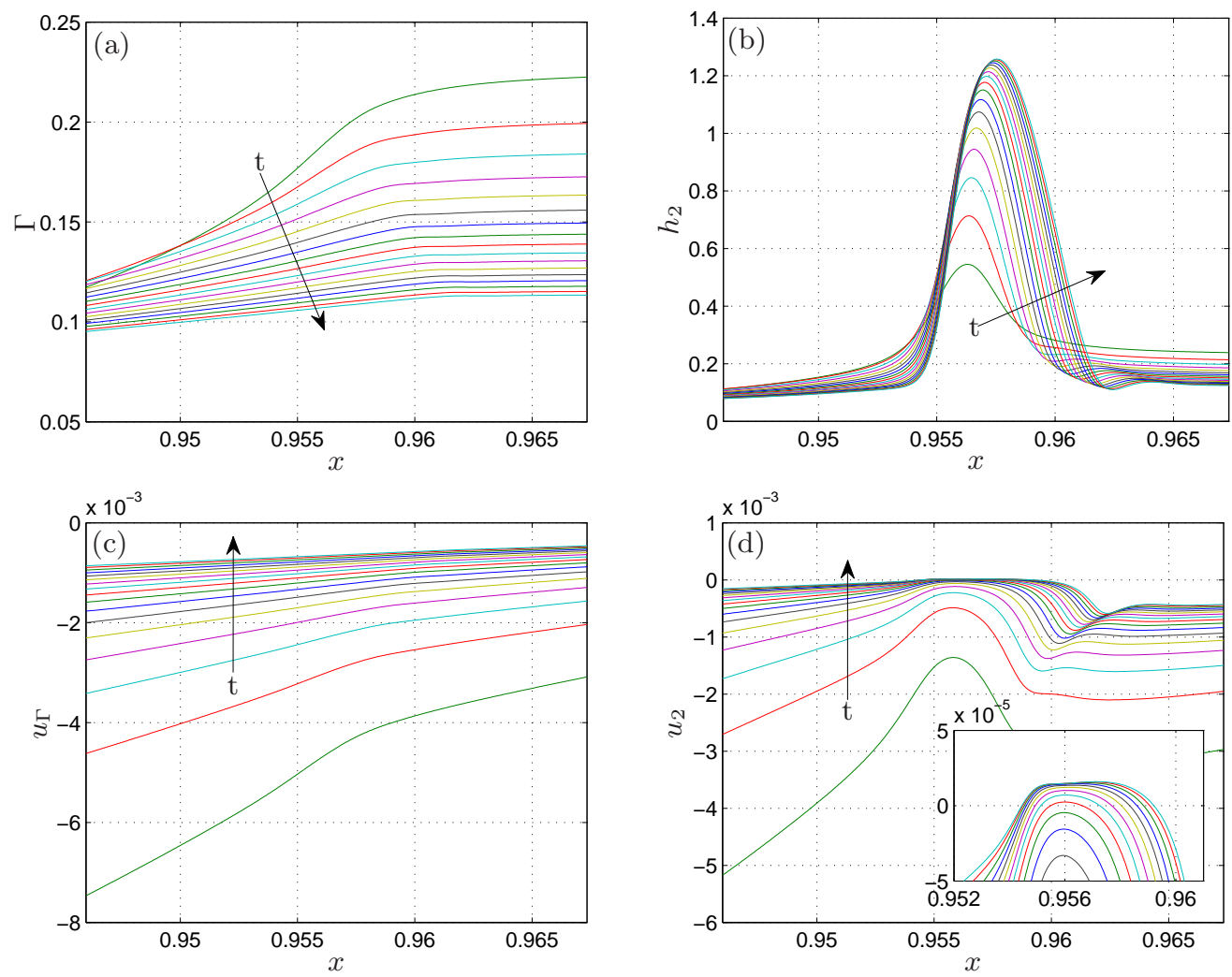

FiguRE 11. Ridge area during the interblink corresponding to the numerical solution shown in figure 4: (a) Polar lipid concentration $\Gamma$. (b) Non-polar lipid layer thickness $h_{2}$. (c) Effective polar lipid layer velocity $u_{\Gamma}=u_{2}-\mathcal{P} \Gamma_{x} / \Gamma$. (d) Non-polar lipid layer velocity $u_{2}$.

that $\bar{u}_{1}$ and $u_{2}$ are given by

$$
\bar{u}_{1}=\frac{h_{1}^{2}}{3}\left(\mathcal{C}_{1} h_{1 x x x}-\mathcal{S}_{1}\right)-\frac{\mathcal{M} h_{1}}{2} \Gamma_{x}, \quad u_{2}=\frac{h_{1}^{2}}{2}\left(\mathcal{C}_{1} h_{1 x x x}-\mathcal{S}_{1}\right)-\mathcal{M} h_{1} \Gamma_{x},
$$

and the model reduces to that presented previously in Aydemir et al. (2011). Applying the boundary conditions on $\bar{u}_{1}$ and $u_{2}$ at each eyelid enable us to get explicit conditions on $h_{1 x x x}$ and $\Gamma_{x}$

$$
\begin{aligned}
& h_{1 x x x}=0, \quad \Gamma_{x}=0, \quad \text { at } \quad x=0, \\
& \mathcal{C}_{1} h_{1 x x x}=6(2 \chi-1) \dot{L}+\mathcal{S}_{1}, \quad \mathcal{M} \Gamma_{x}=2(3 \chi-2) \dot{L}, \quad \text { at } \quad x=L .
\end{aligned}
$$

We note that $\Gamma_{x}>0$ at the upper eyelid.

\subsection{The non-polar lipid layer is very thin, $\delta \ll 1$}

We set $\delta$ to be very small and we throw away the $\delta$ terms from the stress balance $(3.39 d)$ while retaining $\hat{\nu}$ in the evaporation. We find that $\bar{u}_{1}$ and $u_{2}$ are given by

$$
\begin{aligned}
& \bar{u}_{1}=\frac{h_{1}^{2}}{3}\left[(1+\mathcal{T}) \mathcal{C}_{1} h_{1 x x x}-\mathcal{S}_{1}\right]-\frac{\mathcal{M} h_{1}}{2} \Gamma_{x}, \\
& u_{2}=\frac{h_{1}^{2}}{2}\left[(1+\mathcal{T}) \mathcal{C}_{1} h_{1 x x x}-\mathcal{S}_{1}\right]-\mathcal{M} h_{1} \Gamma_{x},
\end{aligned}
$$



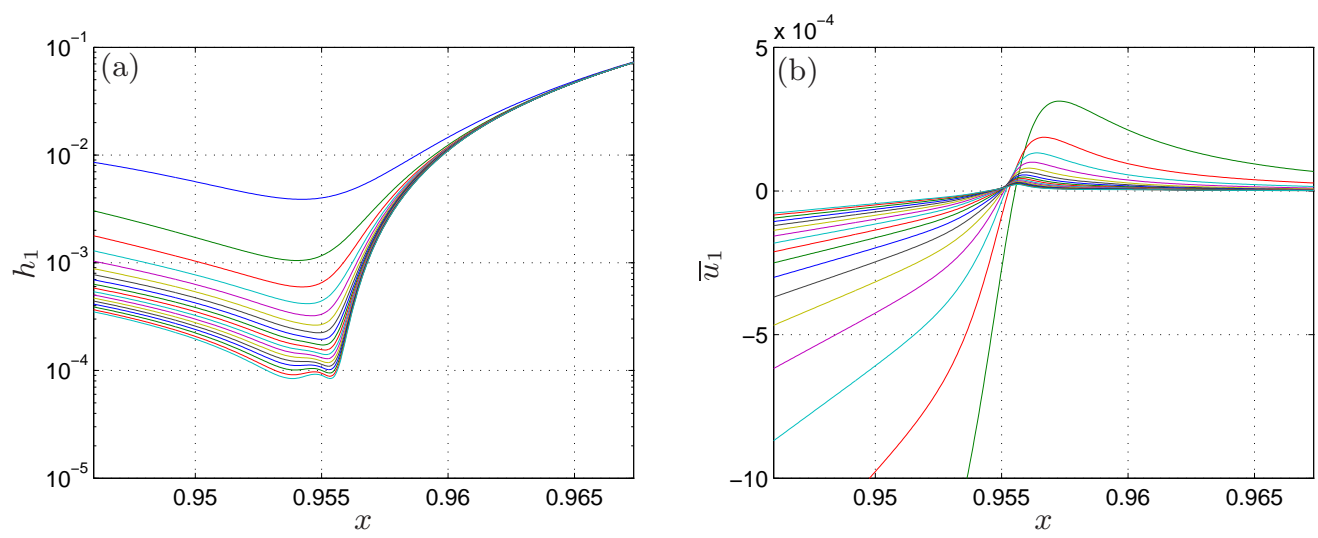

FiguRE 12. Ridge area during the interblink corresponding to the numerical solution shown in figure 4: (a) Aqueous layer thickness $h_{1}$ (logarithmic scale). (b) Aqueous layer velocity $\bar{u}_{1}$.

which are identical to the relationships presented in $(5.1)$ if $(1+\mathcal{T}) \mathcal{C}_{1}$ is replaced by $\mathcal{C}_{1}$. The effect of the non-polar lipid layer is, in this case, to enhance the surface tension of the aqueous layer (as well as providing a barrier to evaporation). With $\mathcal{P}$ nonzero, we cannot apply all the boundary conditions, since setting $\bar{u}_{1}=\chi \dot{L}$ and $u_{2}=\dot{L}$ at $x=L$ and solving for $\left.\Gamma_{x}\right|_{x=L}$ from (5.4a) and (5.4b) gives that $\Gamma_{x}>0$, but setting $\Gamma u_{2}-\mathcal{P} \Gamma_{x}=\Gamma \dot{L}$ as well gives us that $\Gamma_{x}=0$. This indicates the presence of a boundary layer close to the upper eyelid (we have neglected the highest derivative in $(3.39 d)$ ), and that we should satisfy the boundary condition on $\Gamma$ rather than $u_{2}$ at the upper eyelid. We will say more about this boundary layer in appendix A.

\subsection{Not very viscous top layer, $\Upsilon \ll 1$}

When $\Upsilon \ll 1$, we can neglect the viscous term in the longitudinal force balance, while supposing that all the other parameters are $O(1)$. We are then able to solve for $\bar{u}_{1}$ and $u_{2}$ in terms of $h_{1}, h_{2}$ and $\Gamma$. We find that

$$
\begin{aligned}
\bar{u}_{1}= & \frac{h_{1}^{2}}{3}\left[(1+\mathcal{T}) \mathcal{C}_{1} h_{1 x x x}+\delta \mathcal{T} \mathcal{C}_{1} h_{2 x x x}-\mathcal{S}_{1}\right] \\
& -\delta \frac{h_{1} h_{2}}{2}\left[\varrho \mathcal{S}_{1}-\mathcal{T} \mathcal{C}_{1}\left(h_{1 x x x}+\delta h_{2 x x x}\right)\right]-\frac{\mathcal{M} h_{1}}{2} \Gamma_{x}, \\
u_{2}= & \frac{h_{1}^{2}}{2}\left[(1+\mathcal{T}) \mathcal{C}_{1} h_{1 x x x}+\delta \mathcal{T} \mathcal{C}_{1} h_{2 x x x}-\mathcal{S}_{1}\right] \\
& -\delta h_{1} h_{2}\left[\varrho \mathcal{S}_{1}-\mathcal{T} \mathcal{C}_{1}\left(h_{1 x x x}+\delta h_{2 x x x}\right)\right]-\mathcal{M} h_{1} \Gamma_{x} .
\end{aligned}
$$

Despite having neglected the viscous term in $(3.39 d)$, we are able to satisfy the boundary condition on $u_{2}$ and the boundary condition on $\Gamma$ at the upper eyelid. We find that the boundary conditions on the fluxes at the upper eyelid lead to

$$
\begin{aligned}
\Gamma_{x} & =0 \\
\mathcal{C}_{1} h_{1 x x x} & =\frac{2(3 \chi-2) \dot{L}}{\delta h_{2}}+6(2 \chi-1) \dot{L}+\mathcal{S}_{1}(1-\rho), \\
\delta \mathcal{T} \mathcal{C}_{1} h_{2 x x x} & =-\frac{2(1+\mathcal{T})(3 \chi-2) \dot{L}}{\delta h_{2}}-6(2 \chi-1) \mathcal{T} \dot{L}+\mathcal{S}_{1}[(1+\mathcal{T}) \rho-\mathcal{T}],
\end{aligned}
$$

at $x=L$. We note that the limit $\delta \rightarrow 0$ is singular and that we do not recover the "outer" 
boundary conditions from section 5.1 in this limit. Again, this suggests the existence of a boundary layer as $\delta \rightarrow 0$.

\subsection{Very viscous top layer, $\Upsilon \gg 1$}

We suppose that $\Upsilon \gg 1$, divide the longitudinal force balance (3.39d) by $\Upsilon$, and neglect the $1 / \Upsilon$ terms. This means that the surface tension effects in the lipid layer are negligible, and thus we drop the boundary conditions $h_{2 x}=0$ at each eyelid. The model for the non-polar lipid layer then decouples from the rest of the model, and reads:

$$
\begin{aligned}
h_{2 t}+\left(h_{2} u_{2}\right)_{x} & =0 \\
4\left(h_{2} u_{2 x}\right)_{x} & =0,
\end{aligned}
$$

with

$$
h_{2}(x, 0)=1, \quad u_{2}(0, t)=0, \quad u_{2}(L, t)=\dot{L} .
$$

We can see from (5.7) that there is a solution in which $h_{2}$ is only a function of time, namely

$$
h_{2}=\frac{\lambda}{L}, \quad u_{2}=\frac{\dot{L}}{L} x,
$$

and we note in passing that the tension in the non-polar lipid layer is given by $T=\lambda \dot{L} / L^{2}$. Having solved for the velocity in the non-polar lipid layer, $h_{1}$ and $\Gamma$ satisfy

$$
\begin{aligned}
h_{1 t}+\frac{1}{12}\left\{h_{1}^{3}\left[(1+\mathcal{T}) \mathcal{C}_{1} h_{1 x x x}+\delta \mathcal{T} \mathcal{C}_{1} h_{2 x x x}-\mathcal{S}_{1}\right]\right\}_{x}+\frac{\dot{L}}{2 L}\left(x h_{1}\right)_{x} & =0, \\
\Gamma_{t}+\frac{\dot{L}}{L}(x \Gamma)_{x} & =0 .
\end{aligned}
$$

In this parameter limit, the polar lipid layer does not contribute to the evolution of the tear film. We note that, given that $\Gamma$ is also constant at $t=0$, there is also a solution where $\Gamma=\Gamma(t)=\lambda / L$.

Finally, if we suppose that $\mathcal{T}=0$, that is, that we may neglect the influence of the surface tension of the air-lipid interface on the evolution of the aqueous layer, we recover the "uniform stretching" model proposed by Jones et al. (2005).

\subsection{Comparison with "surface viscosity", $\Upsilon \delta \sim O(1), \delta \ll 1, \mathcal{M} \ll 1$}

We now suppose that $\delta$ and $\mathcal{M}$ are very small and that $\Upsilon^{*}=\Upsilon \delta$ is $O(1)$. In this limit, the velocities satisfy

$$
\begin{aligned}
u_{1} & =\frac{1}{2}\left[\mathcal{S}_{1}-(1+\mathcal{T}) \mathcal{C}_{1} h_{1 x x x}\right]\left(y^{2}-h_{1} y\right)+u_{2} \frac{y}{h_{1}}, \\
\bar{u}_{1} & =\frac{h_{1}^{2}}{12}\left[(1+\mathcal{T}) \mathcal{C}_{1} h_{1 x x x}-\mathcal{S}_{1}\right]+\frac{u_{2}}{2} \\
4 \Upsilon^{*}\left(h_{2} u_{2 x}\right)_{x} & =\frac{u_{2}}{h_{1}}-\frac{h_{1}}{2}\left[(1+\mathcal{T}) \mathcal{C}_{1} h_{1 x x x}-\mathcal{S}_{1}\right] .
\end{aligned}
$$

Models for surface viscosity (see e.g. Dey et al. 1997) assume that dilation of the surface causes a change in the surface stress. The simplest model is to assume $\gamma-\gamma_{0}=\Lambda \eta u_{s x}$, where $u_{s}$ is the surface velocity. Thus, the boundary condition (3.23) is replaced by

$$
u_{1 y}=\Lambda\left(\eta u_{s x}\right)_{x},
$$

where $\Lambda=\epsilon \eta_{0} / \mu_{1} L_{o p}$. Taking the derivative with respect to $y$ of $u_{1}$ in $(5.10 a)$ and 
evaluating at the aqueous interface, we find that

$$
\Lambda\left(\eta u_{s x}\right)_{x}=\frac{u_{s}}{h_{1}}-\frac{h_{1}}{2}\left[(1+\mathcal{T}) \mathcal{C}_{1} h_{1 x x x}-\mathcal{S}_{1}\right]
$$

Thus, our model resembles a "surface viscosity" model if we set

$$
\eta(x, t)=\frac{4 \Upsilon^{*} h_{2}(x, t)}{\Lambda} .
$$

\subsection{Physically relevant sublimit}

We recall from table 2 that

$$
\begin{array}{llllrl}
\delta=1.2 \times 10^{-3}, & \Upsilon=4.8 \times 10^{-2}, & \mathcal{C}_{1}=1.1 \times 10^{-2}, & \mathcal{T}=0.4 & \varrho=0.9 \\
\mathcal{E}=9.2 \times 10^{-5}, & \hat{\nu}=3.3 \times 10^{-2}, & \mathcal{P}=5.9 \times 10^{-5}, & \mathcal{S}_{1}=9.4, & \mathcal{M}=0.38
\end{array}
$$

We choose to retain the two evaporation parameters $\mathcal{E}$ and $\hat{\nu}$, despite their sizes, because we are interested in exploring the local evaporative rates. Further, and as in Aydemir et al. (2011), we retain the capillary terms in the model since we expect that capillary forces will dominate in the menisci. We let $\delta \rightarrow 0, \mathcal{P} \rightarrow 0$, and the physically relevant model reads

$$
\begin{aligned}
h_{1 t}+\left(h_{1} \bar{u}_{1}\right)_{x} & =-\mathcal{E} \frac{\hat{\nu}}{\hat{\nu}+h_{2}}, \\
h_{2 t}+\left(h_{2} u_{2}\right)_{x} & =0 \\
\Gamma_{t}+\left(\Gamma u_{2}\right)_{x} & =0,
\end{aligned}
$$

where

$$
\begin{aligned}
& \bar{u}_{1}=\frac{h_{1}^{2}}{3}\left[(1+\mathcal{T}) \mathcal{C}_{1} h_{1 x x x}-\mathcal{S}_{1}\right]-\frac{\mathcal{M} h_{1}}{2} \Gamma_{x}, \\
& u_{2}=\frac{h_{1}^{2}}{2}\left[(1+\mathcal{T}) \mathcal{C}_{1} h_{1 x x x}-\mathcal{S}_{1}\right]-\mathcal{M} h_{1} \Gamma_{x}
\end{aligned}
$$

with

$$
\begin{array}{llll}
h_{1}=1, & h_{1 x x x}=\frac{\mathcal{S}_{1}}{(1+\mathcal{T}) \mathcal{C}_{1}}, & \Gamma_{x}=0, & \text { at } x=0, \\
h_{1}=1, & h_{1 x x x}=\frac{\mathcal{S}_{1}+6(2 \chi-1) \dot{L}}{(1+\mathcal{T}) \mathcal{C}_{1}}, & \Gamma_{x}=\frac{2(3 \chi-2) \dot{L}}{\mathcal{M}}, & \text { at } x=L,
\end{array}
$$

and

$$
\Gamma=1, \quad h_{2}=1, \quad h_{1}=\frac{3 \hat{V}}{2}-\frac{1}{2}+\frac{3}{2}(1-\hat{V})\left(\frac{2 x}{\lambda}-1\right)^{2}, \quad \text { at } \quad t=0 .
$$

This model gives the 'outer' behaviour we expect to see away from the upper eyelid. We note that $\delta \Upsilon \sim \mathcal{P} \ll 1$ and we expect (as we will discuss in appendix A) a small boundary layer to exist near the upper eyelid while the eyelid is moving where the gradient in the concentration of polar lipid, and the first-order correction to the velocity in the non-polar lipid layer, both adjust to zero.

We note that $h_{2}$ and $\Gamma$ satisfy the same equation, initial condition, and boundary conditions (on $u_{2}$ ) and that, comparing figures $9(\mathrm{a}-\mathrm{b})$ and $10(\mathrm{a}-\mathrm{b})$ we see that the profiles for $h_{2}$ and $\Gamma$ are identical when the Péclet number is zero. We explore the relationship between $\Gamma$ and $h_{2}$ in a much simpler paradigm problem in appendix $\mathrm{C}$, and again conclude that $\Gamma=h_{2}$. We thus use the ansatz $\Gamma=h_{2}$ and solve for the unknowns $h_{1}$ and $h_{2}$ 

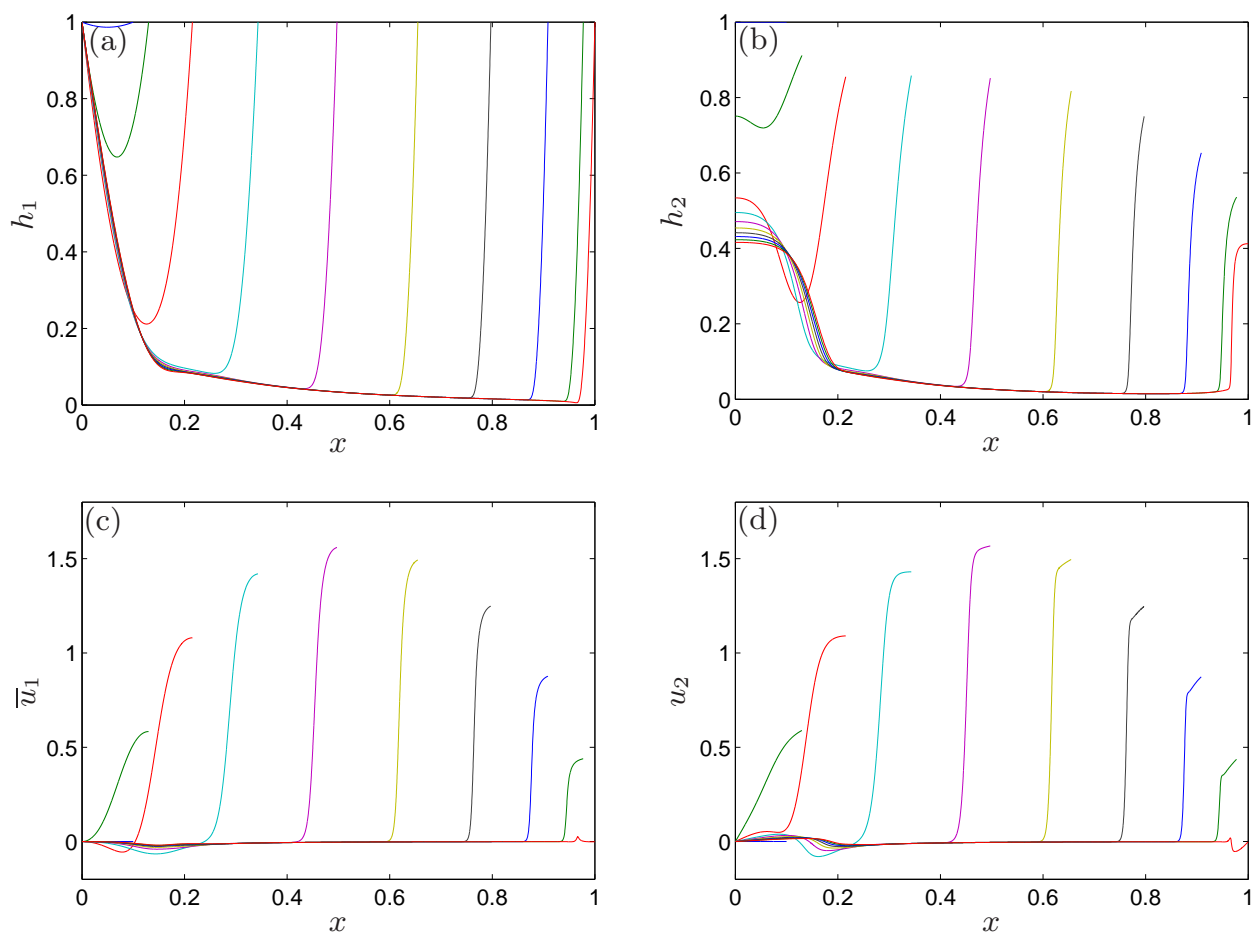

FIGURE 13. Evolution of $h_{1}, h_{2}, \bar{u}_{1}$ and $u_{2}$ in the sublimit (5.15) during the upstroke, for $t=0,0.1, \ldots, 0.9$. The values of the parameters are given in table 2 except for $\delta=\mathcal{P}=0$.

only. We show the solution in figure 13 during the upstroke and in figure 14 during the interblink. Comparing these figures with the corresponding solution of the full model shown in figures 3 and 4, we see that the behaviour is identical, except for the ridges in $h_{2}$, as expected.

\section{Conclusions and future directions}

In this paper we have studied the effect of non-polar lipids on the evolution of the tear film. Our model included the interplay between the aqueous layer, the surface-active polar lipids, and the non-polar lipid layer, and comprised three coupled partial differential equations describing the evolution of the thickness of the aqueous layer, the thickness of the non-polar lipid layer and the concentration of the polar lipids, along with an ordinary differential equation describing the force balance in the non-polar lipid layer. In more detail, the aqueous layer evolves due to a "lubrication flow" while the non-polar lipid layer evolves due to an "extensional flow".

Our numerical simulations of the model exhibit similar aqueous layer and polar lipid behaviour to that seen previously. The non-polar lipid layer thickness has a similar profile to the polar lipid concentration during the upstroke. As the blink evolves, reservoirs of non-polar lipid are formed near the two eyelids joined by a thin layer which stretches over the majority of the eye surface. Thus we conclude that evaporation will be most prevalent away from the eyelids. However, an important difference between the behaviour of the two types of lipid is that the non-polar lipid has a precursor layer that spreads up the upper eyelid during the blink. In the interblink, ridges form near both eyelids which 

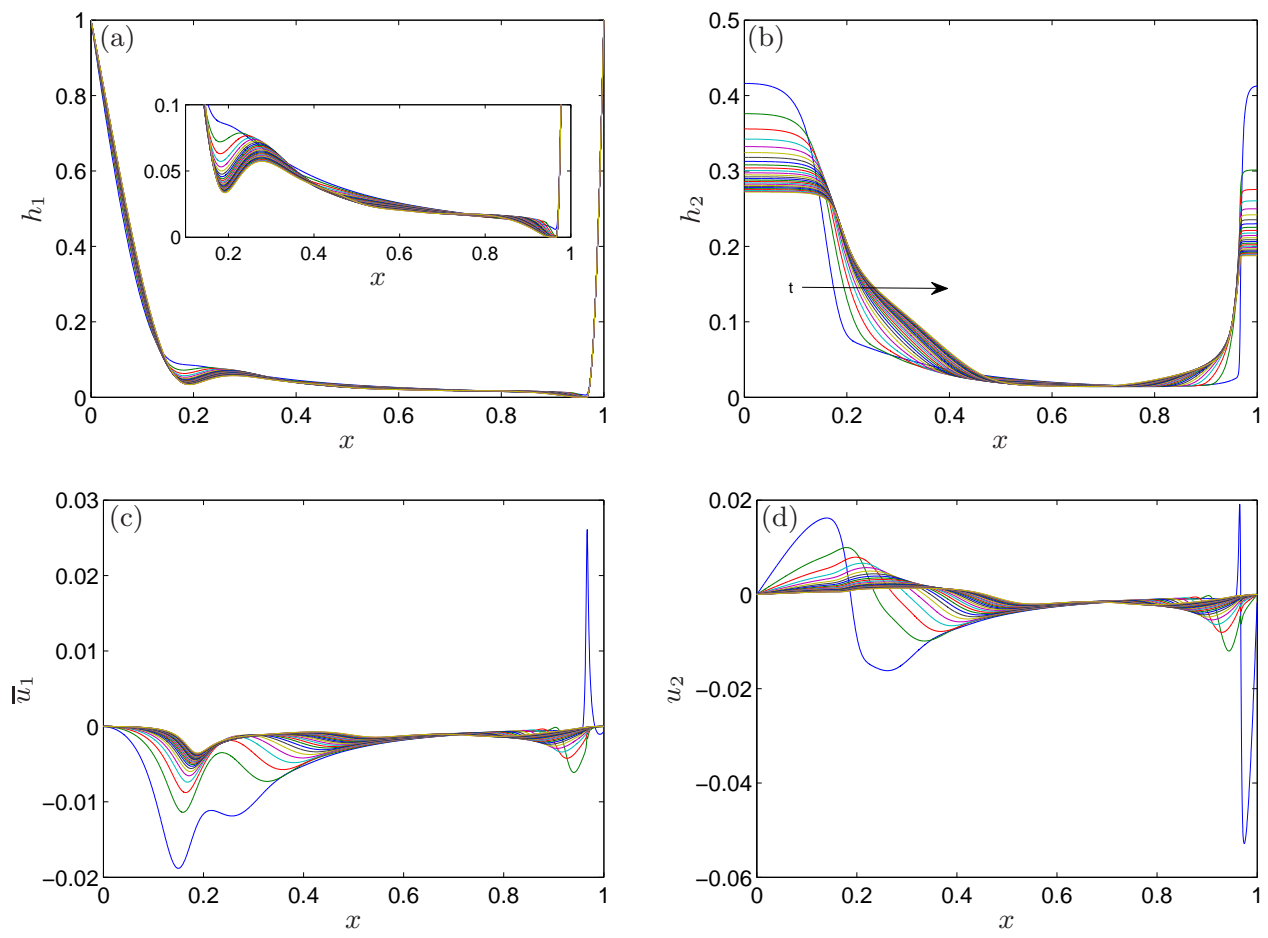

FIGURE 14. Graphs showing the evolution of $h_{1}, h_{2}, \bar{u}_{1}$ and $u_{2}$ in the sublimit (5.15) during the interblink, for $t=0.9,1.9, \ldots, 26.5$. The values of the parameters are given in table 2 except for $\delta=\mathcal{P}=0$.

are co-located near the stagnation point in the flow, while the precursor layer dies away. These ridges are indistinguishable when the overall film thickness is observed (see figure $5)$.

We altered the key model parameters in order to explore the behaviour of the system. Increasing the evaporation rate resulted in reduced minimum tear film thickness as expected. Increasing the Marangoni number enhances the spreading of the non-polar lipid layer and this increased the thickness of the aqueous layer. Increasing the Péclet number reduced the reservoir of polar lipid at the upper eyelid and increased the height of the ridges in the non-polar lipid layer found near the upper and lower eyelids. This local thickening of the non-polar lipid layer may appear counterintuitive, but results from the underlying fluid flow caused, in part, by the diffusion of the polar lipid layer.

Our model for the tear film contains as sublimits previous models derived by other authors. We have identified the parameter scalings required to reduce our model to theirs. For example, by assuming the non-polar lipid layer is very viscous and that the surface tension of the lipid-air interface is negligible, we can reduce the model to the "uniform stretching" model employed by Jones et al. (2005) and other authors. In other sublimits, a boundary layer forms close to the upper eyelid across which the velocity of the non-polar lipid and the gradient of the concentration of polar lipid adjust rapidly. We have demonstrated that matching was possible in several parameter limits.

There are several key assumptions which should be considered more carefully in the future. Firstly, we recall that despite the size of the Reynolds numbers, we dropped inertia completely from our model. We believe that there may be some interesting inertial effects that our current model does not capture and, indeed, including inertia in the non-polar 
lipid layer turns the ODE for the surface velocity (3.39d) into a PDE for $u_{2}$. We intend to examine this limit in the future, and anticipate that the revised model will, in fact, be easier to solve. Secondly, we will look at the boundary condition at the non-polar lipid-eyelid junction. There is an obvious "inner problem" to be considered here, where the free surface adjusts to adopt the appropriate contact angle. We anticipate that this will involve studying a lubrication-style problem where variations up the eyelid are much more dominant than variations perpendicular to the eyelid. Thirdly, our evaporation term should be examined alongside other possibilities, and the resulting flows compared and contrasted. Fourthly, the limit $\Upsilon \ll 1$ presented in section 5.3 warrants further attention. In particular, it would be interesting to examine how the limit $\delta \rightarrow 0$ works in this limiting case. Fifthly, our model does not include the closing phase of the eye. Incorporating this stage would enable us to determine whether the repeated patterns that are observed experimentally in the non-polar lipid layer are a feature of our model. However, to fully answer this question will require us to extend our model to incorporate the third dimension, utilising geometries such as that described in Maki et al. (2010). If our Newtonian setting does not reproduce this behaviour, then an obvious extension would be to assume that the non-polar lipid layer is viscoelastic. Finally, we need to determine whether the ridge formed in the non-polar lipid layer are real biological features or artefacts of the model. Given that they are co-located with the stagnation points on the surface of the aqueous layer, a key question to answer is whether or not these stagnation points are present in the (more accurate) Stokes flow description of the meniscus. This would involve a theoretical exploration along the same lines as that detailed in Zubkov et al. (2013), where the lubrication flow and Stokes flow near an eyelid were shown to be markedly different.

Our major aim is to track the concentration of physiological salts during the blink cycle when the tear film is evolving under the influence of both polar and non-polar lipids. This will combine the work presented in this paper with that described in Zubkov et al. (2012). Such a study should enable us, for the first time, to link increases in tear film osmolarity with deficiencies in the non-polar lipid layer, and thus give valuable insight into evaporative dry eye.

\section{Acknowledgments}

The authors acknowledge many very useful discussions with the OCCAM "Eye Team": R. J. Braun, A. J. Bron, E. A. Gaffney, C. P. Please, J. M. Tiffany and V. S. Zubkov. We especially thank J. A. Carrillo, C. Ortner and T. P. Witelski for helpful discussions on the numerical aspects of this work. This publication is based on work supported by award number KUK-C1-013-04 made by King Abdullah University of Science and Technology (KAUST). M.B. is grateful to St John's College, Oxford for funding in the form of a Junior Research Fellowship.

\section{Appendix A. A note on boundary layers near moving upper eyelids}

We illustrate what happens close to the upper eyelid in three sublimits of our model where we cannot simultaneously satisfy the boundary conditions for the fluxes of polar and non-polar lipid.

\section{A.1. First case}

We begin by considering the case where we neglect gravity $\left(\mathcal{S}_{1}=0\right)$ and surface tension of the upper layer $(\mathcal{T}=0)$ and, as a consequence, we drop the boundary condition $h_{2 x}=0$. 
We assume that $\delta \ll 1$ and $\mathcal{P}, \Upsilon \sim O(1)$. In this situation, the velocities are given by

$$
\begin{aligned}
& \bar{u}_{1}=\frac{h_{1}^{2}}{3} \mathcal{C}_{1} h_{1 x x x}-\frac{\mathcal{M} h_{1}}{2} \Gamma_{x}, \\
& u_{2}=\frac{h_{1}^{2}}{2} \mathcal{C}_{1} h_{1 x x x}-\mathcal{M} h_{1} \Gamma_{x},
\end{aligned}
$$

At $x=L$, we have a contradiction if we try and impose both $u_{2}=\dot{L}$ and $\Gamma u_{2}-\mathcal{P} \Gamma_{x}=$ $\Gamma \dot{L}$, since together these imply that $\Gamma_{x}$ must be zero at $x=L$, and thus we cannot satisfy $\bar{u}_{1}=\chi \dot{L}$ and $u_{2}=\dot{L}$ (we find that $h_{1 x x x}$ must take two different values). This indicates the presence of a boundary layer near $x=L$ in which we must bring back in the derivatives of $u_{2}$ that we have dropped, and over which the velocity of the non-polar lipid layer must adjust. We thus drop the condition $u_{2}=\dot{L}$ from the outer problem, and the boundary conditions to apply on the outer problem at $x=L$ are:

$$
h_{1}=1, \quad \mathcal{C}_{1} h_{1 x x x}=6 \dot{L}\left(\frac{2 \chi-1+\frac{2 \chi \mathcal{P}}{\mathcal{M} \Gamma}}{1+\frac{4 \mathcal{P}}{\mathcal{M} \Gamma}}\right), \quad \mathcal{M} \Gamma_{x}=\frac{(6 \chi-4) \dot{L}}{1+\frac{4 \mathcal{P}}{\mathcal{M} \Gamma}} .
$$

We find that the velocity of the non-polar lipid layer at $x=L$ is

$$
u_{2}=\dot{L}\left(\frac{1+\frac{6 \chi \mathcal{P}}{\mathcal{M} \Gamma}}{1+\frac{4 \mathcal{P}}{\mathcal{M} \Gamma}}\right)>\dot{L}
$$

i.e. it is going too fast.

We scale into the boundary layer close to the upper eyelid by setting $x=L-\sqrt{\delta} \xi$. The equations for the aqueous layer become

$$
h_{1} \bar{u}_{1}=\chi \dot{L}, \quad \bar{u}_{1}=-\frac{h_{1}^{2}}{12} \mathcal{C}_{1} \frac{h_{1 \xi \xi \xi}}{\delta^{(3 / 2)}}+\frac{u_{2}}{2},
$$

which indicate that, at leading order, $h_{1 \xi \xi \xi}=0$. Thus

$$
h_{1}=1+A_{1}(t) \xi+A_{2}(t) \xi^{2} .
$$

In order to successfully match to a finite outer solution, we must pick $A_{1}=A_{2}=0$. Thus, in the boundary layer, the leading-order solutions are

$$
h_{1}=1, \quad \bar{u}_{1}=\chi \dot{L} .
$$

The equations for the non-polar lipid layer become

$$
\begin{aligned}
h_{2} u_{2} & =Q, \\
4 \Upsilon\left(h_{2} u_{2 \xi}\right)_{\xi} & =\frac{1}{h_{1}}\left(4 u_{2}-6 \bar{u}_{1}\right)-\frac{\mathcal{M}}{\sqrt{\delta}} \Gamma_{\xi},
\end{aligned}
$$

where $Q$ is the flux of non-polar lipid into the boundary layer. The polar lipid equation is quasi-steady in the boundary layer, and can be integrated once to give

$$
\Gamma_{\xi}=-\sqrt{\delta} \frac{\Gamma}{\mathcal{P}}\left(u_{2}-\dot{L}\right),
$$

where we have applied the no-flux boundary condition from (3.39f) at the upper eyelid.

Equation (A $6 d$ ) tells us that $\Gamma$ is constant across the layer at leading order, and equal to $\Gamma_{o}$, say. We substitute for $h_{1}, \bar{u}_{1}, h_{2}$ and $\Gamma_{\xi}$ into (A $6 c$ ), and further scale $\xi=2 \sqrt{Q \Upsilon} \zeta$ to remove the $Q$ and $\Upsilon$ dependence, and we find that the equation for $u_{2}$ becomes

$$
\left(\frac{u_{2 \zeta}}{u_{2}}\right)_{\zeta}=\left(4 u_{2}-6 \chi \dot{L}\right)+\frac{\mathcal{M}}{\mathcal{P}} \Gamma_{o}\left(u_{2}-\dot{L}\right),
$$



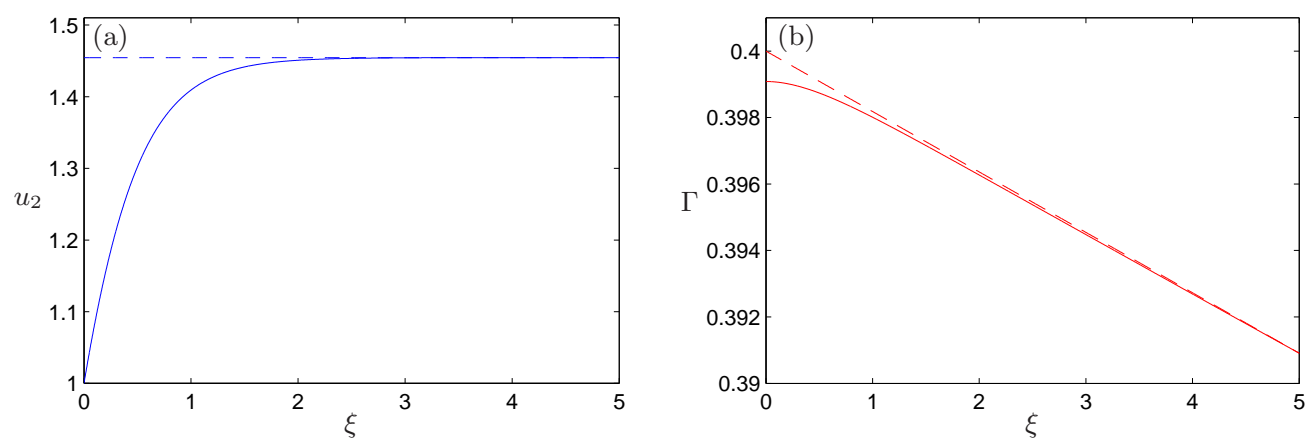

FiguRE 15. Boundary layer solution in the first case (appendix A.1). (a) Solution to (A 7) for $u_{2}$. (b) Solution to $(\mathrm{A} 6 d$ ) for $\Gamma$. The parameters were taken to be: $\chi=1, \mathcal{M}=1, \mathcal{P}=1, \dot{L}=1$, $\delta=10^{-4}$ and $\Gamma_{o}=0.4$. The dashed lines indicate the outer solution.

Across this boundary layer, we expect that $u_{2}$ will decrease from the velocity given in (A 3) to $\dot{L}$, and at the same time the gradient of polar lipid will decrease from the value given in (A 2) to zero. We illustrate the solution to (A 7) for an arbitrarily chosen set of parameters, consistent with this regime, in figure 15 . We assume that the outer solution written in terms of the inner variable is $\Gamma=\Gamma_{o}-(6 \chi-4) \dot{L} \mathcal{P} \xi /\left(\mathcal{M}+4 \mathcal{P} / \Gamma_{o}\right)$. We see that the concentration of polar lipid only deviates slightly from the outer solution, as expected.

\section{A.2. Second case}

In the case where $\mathcal{P}$ is small, say the same size as $\delta \Upsilon$, at leading order the boundary conditions for the flux of polar and non-polar lipid collapse to the same condition, namely $u_{2}=\dot{L}$ (but recall that this means that $\Gamma_{x}>0$ at $x=L$ ). We solve the outer problem assuming that this condition holds at $x=L$ while, close to $x=L$, we scale $x=L-\mathcal{P} \xi$. We find that the leading-order solutions for $h_{1}$ and $\bar{u}_{1}$ are identical to those presented in (A $6 a$ ), while the other variables satisfy

$$
\begin{aligned}
h_{2} u_{2} & =Q, \\
\left(u_{2} \Gamma+\Gamma_{\xi}\right)_{\xi} & =0, \\
4 B\left(h_{2} u_{2 \xi}\right)_{\xi} & =\frac{\mathcal{P}}{h_{1}}\left(4 u_{2}-6 u_{1}\right)-\mathcal{M} \Gamma_{\xi},
\end{aligned}
$$

where $B=\delta \Upsilon / \mathcal{P}$. Since $u_{2}=\dot{L}$ at $x=L$ and at the edge of the boundary layer (at leading order in $\mathcal{P}$ ), we set $u_{2}=\dot{L}+\mathcal{P} u_{2}^{(1)}$. Equation (A 8c) then yields that $\Gamma_{\xi}=0$ to leading order. Thus we set $\Gamma=\Gamma_{o}+\mathcal{P} \Gamma^{(1)}$ and find that

$$
\begin{aligned}
\Gamma_{\xi}^{(1)} & =\left(\Gamma^{(1)}(0)-\Gamma^{(1)}\right) \dot{L}-\Gamma_{o} u_{2}^{(1)}, \\
4 B\left(h_{2} u_{2 \xi}^{(1)}\right)_{\xi} & =(4-6 \chi) \dot{L}-\mathcal{M} \Gamma_{\xi}^{(1)},
\end{aligned}
$$

where recall that the superscripts $(1)$ indicate the $O(\mathcal{P})$ terms in the asymptotic expansion. Remembering that $h_{2}=Q / \dot{L}=$ constant, we can combine these two equations to give

$$
\Gamma_{\xi \xi \xi}^{(1)}+\dot{L} \Gamma_{\xi \xi}^{(1)}-\frac{\mathcal{M} \Gamma_{o}}{4 B h_{2}} \Gamma_{\xi}^{(1)}=\frac{(6 \chi-4) \Gamma_{o} \dot{L}}{4 B h_{2}}
$$



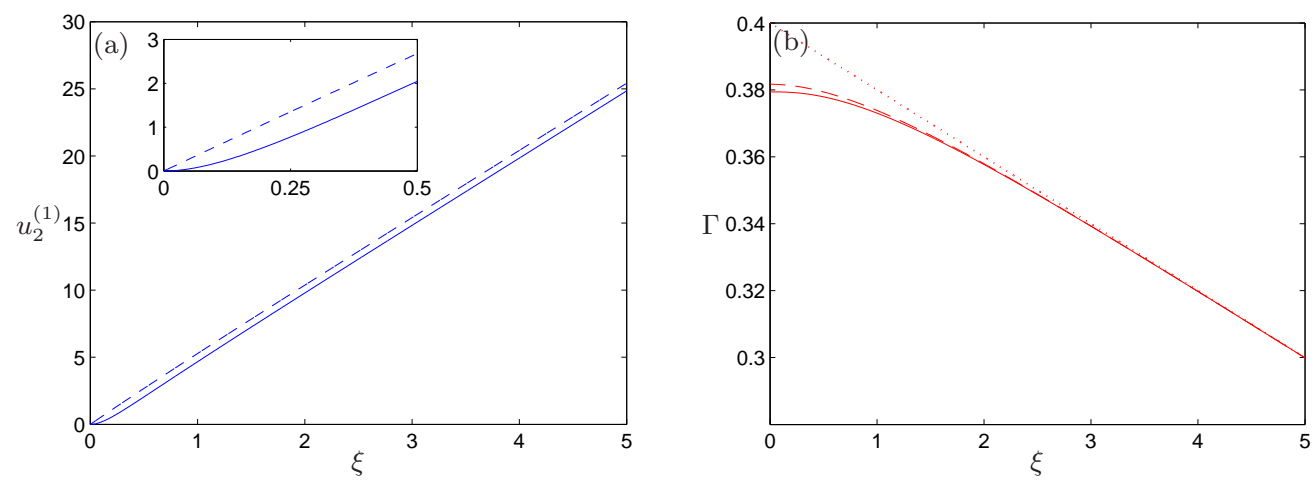

Figure 16. Boundary layer solutions for $u_{2}$ and $\Gamma$ in the second and third cases. (a) Inner velocity $u_{2}^{(1)}$, given by (A 12) (dashed line) and (A 20) (solid line). (b) Polar lipid concentration $\Gamma$, where $\Gamma^{(1)}$ is given by (A 11) (dashed line) and (A 19) (solid line). The outer solution for $\Gamma$ is shown by the dotted line. Other parameters were set to one, except for $\Gamma_{o}=0.4, \mathcal{P}=\delta=0.01$.

The solution to (A 10) that does not blow up exponentially at infinity and satisfies the correct boundary condition at $\xi=0$ is

$$
\Gamma^{(1)}=\frac{(6 \chi-4) \dot{L}}{\mathcal{M}}\left(A_{1}-\xi+\frac{1}{\lambda} e^{\lambda \xi}\right)
$$

where $\lambda=-\frac{\dot{L}}{2}\left(1+\sqrt{1+\frac{\mathcal{M} \Gamma_{o}}{B h_{2} \dot{L}^{2}}}\right)$ and $A_{1}$ is determined by matching with the outer problem. We use this solution and the appropriate boundary conditions to solve (A $9 b$ ) to find

$$
u_{2}^{(1)}=\frac{(6 \chi-4) \dot{L}}{4 B h_{2} \lambda^{2}}\left(1+\frac{4 B h_{2} \dot{L} \lambda^{2}}{\mathcal{M} \Gamma_{o}} \xi-e^{\lambda \xi}\right) .
$$

In this case, both $\Gamma^{(1)}$ and $u_{2}^{(1)}$ are linear at the edge of the boundary layer, and matching with the outer solution shows that we expect to get positive gradients for both $\Gamma$ and $u_{2}$ as we get close the upper eyelid. We plot this solution (with a dashed line) in figure 16.

\section{A.3. Third case}

Finally, we reintroduce the surface tension $\mathcal{T}=O(1)$ of the upper layer in this case where $\mathcal{P}$ is small, and remember that we then also have to satisfy $h_{2 x}=0$ at $x=L$. We follow the same recipe as before, setting $B \mathcal{P}=\delta \Upsilon$ and scaling $x=L-\mathcal{P} \xi$. The boundary layer 
model reads

$$
\begin{aligned}
\left(h_{1} \bar{u}_{1}\right)_{\xi}= & 0, \\
\left(h_{2} u_{2}\right)_{\xi}= & 0, \\
\bar{u}_{1}= & -\frac{\mathcal{C}_{1} h_{1}^{2}}{12 \mathcal{P}^{3}}\left((1+\mathcal{T}) h_{1 \xi \xi \xi}+\delta \mathcal{T} h_{2 \xi \xi \xi}\right)+\frac{u_{2}}{2}, \\
\left(\Gamma u_{2}+\Gamma_{\xi}\right)_{\xi}= & 0, \\
4 \frac{B}{\mathcal{P}}\left(h_{2} u_{2 \xi}\right)_{\xi}= & \frac{\left(4 u_{2}-6 \bar{u}_{1}\right)}{h_{1}}-\frac{6 \delta \mathcal{T} h_{2}}{h_{1}^{2}(1+\mathcal{T})}\left(2 \bar{u}_{1}-u_{2}\right) \\
& +\frac{\delta^{2} \mathcal{C}_{1} \mathcal{T}}{\mathcal{P}^{3}(1+\mathcal{T})} h_{2} h_{2 \xi \xi \xi}-\frac{\mathcal{M}}{\mathcal{P}} \Gamma_{\xi} .
\end{aligned}
$$

As previously, (A $6 a$ ) holds, and we again set $u_{2}=\dot{L}+\mathcal{P} u_{2}^{(1)}$. In this case, we will also need to expand $h_{2}(\xi)=h_{2}+\mathcal{P} h_{2}^{(1)}$, where $h_{2}=$ constant. Equation (A 13e) then becomes

$$
4 B h_{2} u_{2 \xi \xi}^{(1)}=(4-6 \chi) \dot{L}-\mathcal{P} \frac{6 B h_{2} \mathcal{T}}{\Upsilon(1+\mathcal{T})}(2 \chi-1) \dot{L}+\frac{B^{2} \mathcal{T} \mathcal{C}_{1} h_{2}}{\Upsilon^{2}(1+\mathcal{T})} h_{2 \xi \xi \xi}^{(1)}-\frac{\mathcal{M}}{\mathcal{P}} \Gamma_{\xi},
$$

which tells us that $\Gamma=\Gamma_{o}=$ constant to leading order, and thus $\Gamma^{(1)}$ satisfies (A $\left.9 a\right)$ as before. We now have to relate $h_{2}^{(1)}$ and $u_{2}^{(1)}$, which we do using the $O(\mathcal{P})$ version of (A 13b), which gives us that

$$
h_{2}^{(1)}=\frac{Q^{(1)}-h_{2} u_{2}^{(1)}}{\dot{L}}
$$

We rearrange $(\mathrm{A} 9 a)$ to get $u_{2}^{(1)}$ as a function of $\Gamma^{(1)}$ and we can therefore re-write (A 14) as an equation solely for $\Gamma^{(1)}$, which reads

$$
\frac{B^{2} \mathcal{T} \mathcal{C}_{1} h_{2}^{2}}{\Upsilon^{2}(1+\mathcal{T}) \dot{L} \Gamma_{o}}\left(\Gamma_{\xi}^{(1)}+\dot{L} \Gamma^{(1)}\right)_{\xi \xi \xi}+\frac{4 B h_{2}}{\Gamma_{o}}\left(\Gamma_{\xi}^{(1)}+\dot{L} \Gamma^{(1)}\right)_{\xi \xi}=\mathcal{M} \Gamma_{\xi}^{(1)}-(4-6 \chi) \dot{L}
$$

We seek a solution of the form $\Gamma^{(1)}=a_{0} \xi+a_{1} e^{\lambda \xi}$, where $a_{0}=(4-6 \chi) \dot{L} / \mathcal{M}$, and we find that either $\lambda=0$ or

$$
\lambda^{3}+\lambda^{2}(1+a) \dot{L}+\lambda a \dot{L}^{2}-b=0
$$

where

$$
a=\frac{4(1+\mathcal{T}) \Upsilon^{2}}{h_{2} \mathcal{T} \mathcal{C}_{1} B}, \quad b=\frac{\mathcal{M}^{2}(1+\mathcal{T}) \dot{L} \Gamma_{o}}{B^{2} \mathcal{T} \mathcal{C}_{1} h_{2}^{2}}
$$

Equation (A 17) has at least one positive root (and thus we set the constant of integration corresponding to this root to zero). Writing $a_{1}=a /(1+a)^{2}$ and $b_{1}=b /(1+a)^{3} \dot{L}^{3}$, we find that

(i) if $a_{1}>1 / 3$, there is only one (positive) root.

(ii) if $a_{1}<1 / 3, b_{1}<\left[2-9 a_{1}+\sqrt{1-3 a_{1}}\left(2-6 a_{1}\right)\right] / 27$, then there are two real negative roots.

(iii) if $a_{1}<1 / 3, b_{1}>\left[2-9 a_{1}+\sqrt{1-3 a_{1}}\left(2-6 a_{1}\right)\right] / 27$, then the roots are a conjugate pair with negative real part. 
In the second case (ii), the solutions that satisfy the boundary conditions $\Gamma_{\xi}=0$ and $h_{2 \xi}=0$ at $\xi=0$ are:

$$
\begin{aligned}
\Gamma^{(1)}=\frac{(4-6 \chi) \dot{L}}{\mathcal{M}} & {\left[A_{1}+\xi-\frac{\lambda_{2}}{\lambda_{1}\left(\lambda_{2}-\lambda_{1}\right)} e^{\lambda_{1} \xi}+\frac{\lambda_{1}}{\lambda_{2}\left(\lambda_{2}-\lambda_{1}\right)} e^{\lambda_{2} \xi}\right], } \\
u_{2}^{(1)}=\frac{(4-6 \chi) \dot{L}}{\mathcal{M} \Gamma_{o}} & {\left[\frac{\lambda_{2}}{\lambda_{2}-\lambda_{1}}\left(1+\frac{\dot{L}}{\lambda_{1}}\right) e^{\lambda_{1} \xi}-\frac{\lambda_{1}}{\lambda_{2}-\lambda_{1}}\left(1+\frac{\dot{L}}{\lambda_{2}}\right) e^{\lambda_{2} \xi}\right.} \\
& \left.-\frac{\lambda_{1}+\lambda_{2}}{\lambda_{1} \lambda_{2}} \dot{L}-\dot{L} \xi-1\right],
\end{aligned}
$$

where $\lambda_{1,2}$ are the appropriate roots of (A 17) (and there are similar expressions for the final case (iii)). We compare these solutions with the $\mathcal{T}=0$ solutions given in (A 11) and (A 12) in figure 16. We can see that the presence of the surface tension term makes almost no difference to the solution for $\Gamma^{(1)}$, but makes a reasonable difference to the solution for $u_{2}^{(1)}$, bending the solution away from the surface-tension-free case.

\section{Appendix B. Numerical convergence}

In figure 17 we plot the absolute error in $h_{1}, \Gamma$ and $h_{2}$ as we increase the number of spatial grid points $N=2^{n}$ in the discretisation of the system (3.39). The left column shows how the error evolves in time (error shown is error with respect to the solution with the highest resolution with $n=14$, equivalent to $N=16384$ ). We see that the error decreases as $n$ increases. In the right column we show the convergence plots at three different times, and the curve $10^{-2 n}$ to show the second-order convergence.

\section{Appendix C. A paradigm problem}

The model presented in section 5.6 is complicated and it is helpful to consider a simple paradigm for the interblink that captures the essential behaviour of $u_{2}$, that is, it depends on $\Gamma_{x}$ and changes sign somewhere in the domain (see figure 4(f) for example). Our aim is to examine the difference between $h_{2}$ and $\Gamma$. For convenience we pick $u_{2}=\alpha x-\Gamma_{x}$ and ignore the aqueous layer completely. The paradigm model is then

$$
\begin{aligned}
h_{2 t}+\left(h_{2} u_{2}\right)_{x} & =0, \\
\Gamma_{t}+\left(\Gamma u_{2}\right)_{x} & =0, \\
u_{2} & =\alpha x-\Gamma_{x},
\end{aligned}
$$

with the following initial and boundary conditions

$$
\begin{aligned}
& h_{2}=\Gamma=1+e^{-3 x^{2}}, \quad \text { at } \quad t=0, \\
& \Gamma_{x}=\alpha x, \quad \text { at } \quad x=0,1 \text {. }
\end{aligned}
$$

We solve this problem numerically using Chebfun (Trefethen et al. 2011) in MATLAB. In figure 18(a) we plot the solution for $h_{2}$ and $\Gamma$, and in figure 18(b) we see how the velocity field $u_{2}(x, t)$ evolves. We see that the two solutions for $h_{2}$ and $\Gamma$ are indistinguishable for this given velocity field. This gives us confidence in the numerical procedure chosen to solve the system (5.15), namely setting $h_{2}=\Gamma$ when $\mathcal{P}=\delta=0$.

\section{REFERENCES}



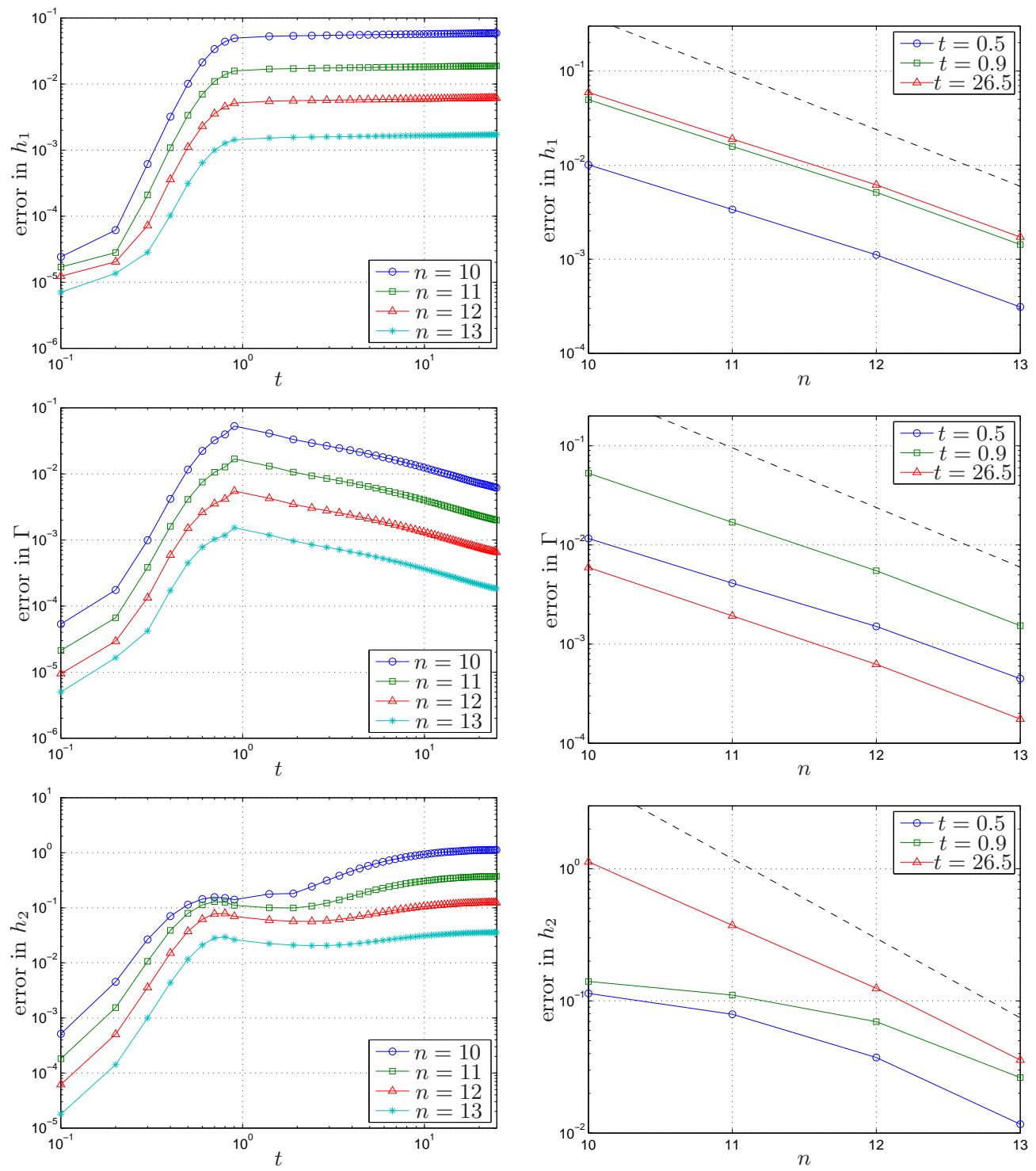

Figure 17. Absolute error in $h_{1}, \Gamma$ and $h_{2}$ in (3.39) as a function of time and the number of spatial grid points $N=2^{n}$, for $n=10,11,12,13$ using the $n=14$ solution as reference. (Left column) Evolution during the upstroke and interblink, for times $t=0,0.1, \ldots, 0.9,1.9, \ldots, 26.5$. (Right column) Convergence plots at three different times, showing the curve $10^{-2 n}$ (dashed).

Aydemir, E., Breward, C. J. W. \& Witelski, T. P. 2011 The effect of polar lipids on tear film dynamics. B. Math. Biol. 73 (6), 1171-1201.

Berger, Robert E \& Corrsin, Stanley 1974 A surface tension gradient mechanism for driving the pre-corneal tear film after a blink. Journal of Biomechanics 7 (3), 225-238.

Berke, A. \& Mueller, S. 1996 Einfluss des lidschlages auf die kontaktlinse und die zugrundeliegenden kräfte. Die Kontaktlinse 1, 17-26.

Berke, A. \& Mueller, S. 1998 The kinetics of lid motion and its effects on the tear film. In Lacrimal Gland, Tear Film, and Dry Eye Syndromes 2: Basic Science and Clinical Relevance (ed. D. A. Sullivan, D. A. Dartt \& M. A. Meneray), Advances in Experimental Medicine and Biology, vol. 438, pp. 417-424. Plenum Press. 

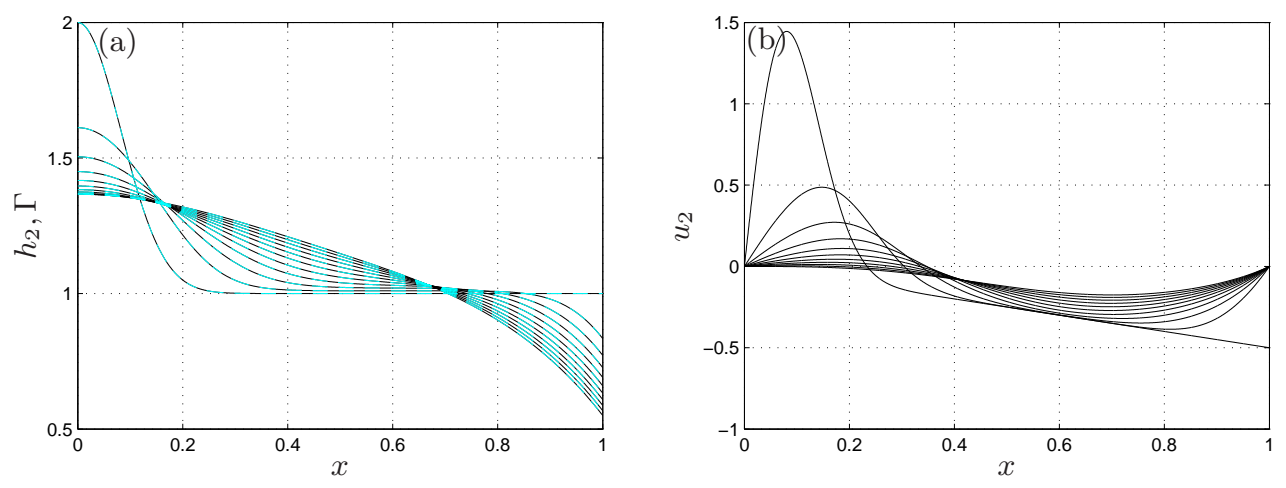

Figure 18. Solution of the paradigm model (C 1) with $\alpha=-0.5$ : (a) Curves for $h_{2}$ (in cyan (light grey) dashed lines) and $\Gamma$ (in black solid lines) are indistinguishable. (b) Velocity field $u_{2}$ changing sign.

Braun, R. J. 2012 Dynamics of the tear film. Annu. Rev. Fluid Mech. 44, 267-297.

Braun, R. J. \& Fitt, A. D. 2003 Modelling drainage of the precorneal tear film after a blink. Math. Med. Biol. 20 (1), 1-28.

Braun, R. J. \& King-Smith, P. E. 2007 Model problems for the tear film in a blink cycle: single-equation models. J. Fluid Mech. 586, 465-490.

Bron, A. J., Tiffany, J. M., Gouveia, S. M., Yokoi, N. \& Voon, L. W. 2004 Functional aspects of the tear film lipid layer. Exp. Eye Res. 78 (3), 347-360.

Chen, J., Green-Church, K. B. \& Nichols, K. K. 2010 Shotgun lipidomic analysis of human meibomian gland secretions with electrospray ionization tandem mass spectrometry. Invest. Ophth. Vis. Sci. 51 (12), 6220-6231.

Dey, D., Boulton-Stone, J. M., Emery, A. N. \& Blake, J. R. 1997 Experimental comparisons with a numerical model of surfactant effects on the burst of a single bubble. Chem. Eng. Sci. 52 (16), 2769-2783.

Dieckow, J. \& Argüeso, P. 2012 The human tear film. In Ocular Surface: Anatomy and Physiology, Disorders and Therapeutic Care (ed. R. Martin Herranz \& R. M. Corrales Herran), chap. 2. CRC Press.

Heryudono, A., Braun, R. J., Driscoll, T. A., Maki, K. L., Cook, L. P. \& King-Smith, P. E. 2007 Single-equation models for the tear film in a blink cycle: realistic lid motion. Math. Med. Biol. 24 (4), 347-377.

Holly, F. J. \& Lemp, M. A. 1977 Tear physiology and dry eyes. Surv. Ophthalmol. 22 (2), 69-87.

Johnson, M. E. \& Murphy, P. J. 2004 Changes in the tear film and ocular surface from dry eye syndrome. Prog. Retin. Eye Res. 23 (4), 449-474.

Jones, M. B., Fulford, G. R., Please, C. P., McElwain, D. L. S. \& Collins, M. J. 2008 Elastohydrodynamics of the eyelid wiper. B. Math Biol. 70 (2), 323-343.

Jones, M. B., McElwain, D. L. S., Fulford, G. R., Collins, M. J. \& Roberts, A. P. 2006 The effect of the lipid layer on tear film behaviour. B. Math Biol. 68 (6), 1355-1381.

Jones, M. B., Please, C. P., Mcelwain, D. L. S., Fulford, G. R., Roberts, A. P. \& Collins, M. J. 2005 Dynamics of tear film deposition and draining. Math. Med. Biol. 22 (3), 265-288.

Kaufman, P. L., Alm, A. \& Adler, F. H. 2003 Adler's physiology of the eye : clinical application, 10th edn. St. Louis : Mosby.

King-Smith, P. E., Fink, B., Hill, R., Koelling, K. \& Tiffany, J. 2004 The thickness of the tear film. Curr. Eye Res. 29 (4-5), 357-368.

Lemp, M. A. 2007 The definition and classification of dry eye disease: report of the definition and classification subcommittee of the international dry eye workshop (2007). Ocul. Surf. 5 (2), 75-92. 
Li, L. \& Braun, R. J. 2012 A model for the human tear film with heating from within the eye. Phys. Fluids 24 (6), 062103-062103-19.

Liu, Haixia, Begley, Carolyn, Chen, Minhua, Bradley, Arthur, Bonanno, Joseph, McNamara, Nancy A, Nelson, J Daniel \& Simpson, Trefford 2009 A link between tear instability and hyperosmolarity in dry eye. Investigative Ophthalmology \& Visual Science 50 (8), 3671-3679.

MacDonald, E. A. \& Maurice, D. M. 1991 The kinetics of tear fluid under the lower lid. Exp. Eye Res. 53 (4), 421-425.

Maki, K. L., Braun, R. J., Driscoll, T. A. \& King-Smith, P. E. 2008 An overset grid method for the study of reflex tearing. Math. Med. Biol. 25 (3), 187-214.

Maki, K. L., Braun, R. J., Ucciferro, P., Henshaw, W. D. \& King-Smith, P. E. 2010 Tear film dynamics on an eye-shaped domain. part 2. flux boundary conditions. J. Fluid Mech. 647, 361-390.

Matar, O. K., Craster, R. V. \& Warner, M. R. E. 2002 Surfactant transport on highly viscous surface films. J. Fluid Mech. 466, 85-111.

McCulley, J. P. \& Shine, W. E. 1997 A compositional based model for the tear film lipid layer. T. Am. Ophthal. Soc. 95, 79-93.

McCulley, J. P. \& Shine, W. E. 2004 The lipid layer of tears: dependent on meibomian gland function. Exp. Eye Res. 78 (3), 361-365.

Mudgil, P. \& Millar, T. J. 2011 Surfactant properties of human meibomian lipids. Invest. Ophth. Vis. Sci. 52 (3), 1661-1670.

Nagyova, B. \& Tiffany, J. M. 1999 Components responsible for the surface tension of human tears. Curr. Eye Res. 19 (1), 4-11.

Nichols, J. J., Mitchell, G. L. \& King-Smith, P. E. 2005 Thinning rate of the precorneal and prelens tear films. Invest. Ophth. Vis. Sci. 46 (7), 2353-2361.

Nicolaides, N., Kaitaranta, J. K., Rawdah, T. N., Macy, J. I., Boswell, F. M. \& Smith, R. E. 1981 Meibomian gland studies: comparison of steer and human lipids. Invest. Ophth. Vis. Sci. 20 (4), 522-536.

Ockendon, H. \& OCKendon, J. R. 1995 Viscous flow, , vol. 13. Cambridge University Press.

Peng, C.-C., Cerretani, C., Braun, R. J. \& Radke, C. J. 2014 Evaporation-driven instability of the precorneal tear film. Adv. Colloid Interfac. 206, 250-264.

Tiffany, J. M. 1978 Individual variations in human meibomian lipid composition. Exp. Eye Res. 27 (3), 289-300.

Tiffany, J. M. 2009 Private Communication.

Tiffany, J. M. \& DART, J. K. G. 1981 Normal and abnormal functions of meibomian secretion. Roy. Soc. Med. Int. Congr. Symp. Ser. 40, 1061-1064.

Trefethen, L. N. et al. 2011 Chebfun Version 4.2. The Chebfun Development Team, http://www. chebfun.org/.

WolfF, E. 1946 The muco-cutaneous junction of the lid-margin and the distribution of the tear fluid. T. Ophthal. Soc. U.K. 66, 291-308.

Wong, H., Fatt, I. \& Radke, C. J. 1996 Deposition and thinning of the human tear film. J. Colloid Interf. Sci. 184 (1), 44-51.

Yokoi, N., Yamada, H., Mizukusa, Y., Bron, A. J., Tiffany, J. M., Kato, T. \& KiNoshitA, S. 2008 Rheology of tear film lipid layer spread in normal and aqueous teardeficient dry eyes. Invest. Ophth. Vis. Sci. 49 (12), 5319-5324.

Zubkov, V. S., Breward, C. J. W. \& Gaffney, E. A. 2012 Coupling fluid and solute dynamics within the ocular surface tear film: A modelling study of black line osmolarity. Bull. Math. Biol. 74 (9), 2062-2093.

Zubkov, V. S., Breward, C. J. W. \& Gaffney, E. A. 2013 Meniscal tear film fluid dynamics near Marxs line. Bull. Math. Biol. 75 (9), 1524-1543. 